NASA Contractor Report 187604

ICASE Report No. 91-58

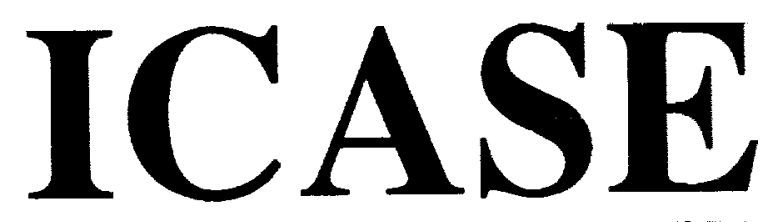

\title{
THE ENERGY DECAY IN SELF-PRESERVING ISOTROPIC TURBULENCE REVISITED
}

Charles G. Speziale Peter S. Bernard

Contract No. NAS1-18605

July 1991

Institute for Computer Applications in Science and Engineering NASA Langley Research Center

Hampton, Virginia 23665-5225

Operated by the Universities Space Research Association

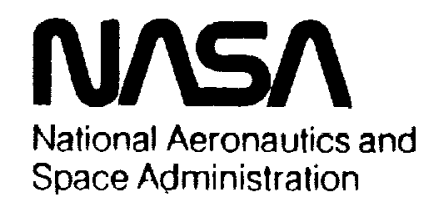

$\begin{array}{ll}n & \\ 0 & n \\ 1 & 0 \\ 1 & 0 \\ 2 & 50\end{array}$

$z$

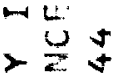

$>2$

$\underset{5}{1}$

$0 \widetilde{0}$

$\therefore$ un

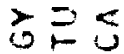

$\alpha$

$>1$

لـ

E +

I

$I \pm$

$\rightarrow x$

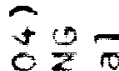

$02 \pi$

$r>-$

$\infty x !$

$\longrightarrow 4$

1 แ $D$

c. ㄴ. U

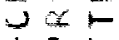

$10 \mathrm{~B}$

$<1$ in

$4 \pm$

$2 \frac{1}{2}$

Space Administration

$\underline{1}$ 
$\Rightarrow x=-6$ 


\title{
THE ENERGY DECAY IN SELF-PRESERVING ISOTROPIC TURBULENCE REVISITED
}

\author{
Charles G. Speziale* \\ Institute for Computer Applications in Science and Engineering, \\ NASA Langley Research Center, \\ Hampton, Virginia 23665 \\ Peter S. Bernard \\ Department of Mechanical Engineering, \\ University of Maryland, \\ College Park, Maryland 20742
}

\begin{abstract}
The assumption of self-preservation allows for an analytical determination of the energy decay in isotropic turbulence. Batchelor (1948), who was the first to carry out a detailed study of this problem, based his analysis on the assumption that the Loitsianskii integral is a dynamic invariant - a widely accepted hypothesis that was later discovered to be invalid. Nonetheless, it appears that the self-preserving isotropic decay problem has never been reinvestigated in depth subsequent to this earlier work. In the present paper such an analysis is carried out, yielding a much more complete picture of selfpreserving isotropic turbulence. It is proven rigorously that complete self-preserving isotropic turbulence admits two general types of asymptotic solutions: one where the turbulent kinetic energy $K \sim t^{-1}$ and one where $K \sim t^{-\alpha}$ with an exponent $\alpha>1$ that is determined explicitly by the initial conditions. By a fixed point analysis and numerical integration of the exact one-point equations, it is demonstrated that the $K \sim t^{-1}$ power law decay is the asymptotically consistent high-Reynolds-number solution; the $K \sim t^{-\alpha}$ decay law is only achieved in the limit as $t \rightarrow \infty$ and the turbulence Reynolds number vanishes. Arguments are provided which indicate that a $K \sim t^{-1}$ power law decay is the asymptotic state toward which a complete self-preserving isotropic turbulence is driven at high Reynolds numbers in order to resolve the imbalance between vortex stretching and viscous diffusion. Unlike in previous studies, the asymptotic approach to a self-preserving state is investigated which uncovers some surprising results.
\end{abstract}

* Research supported by the National Aeronautics and Space Administration under NASA Contract No. NAS1-18605 while the author was in residence at the Institute for Computer Applications in Science and Engineering (ICASE), NASA Langley Research Center, Hampton, VA 23665-5225. 


\section{INTRODUCTION}

Despite the fact that isotropic turbulence constitutes the simplest type of turbulent flow, it is still not possible to render the problem analytically tractable without the introduction of additional hypotheses. The idealization of self-preservation - wherein the two-point double and triple longitudinal velocity correlations are assumed to admit self-similar solutions with respect to a single length scale $L(t)$ - has served as a useful hypothesis since its introduction by von Karman and Howarth (1938). In another classic paper that followed, Batchelor (1948) studied the energy decay in self-preserving isotropic turbulence in considerable detail. He concluded that the only complete self-preserving solutions that were internally consistent existed at low turbulence Reynolds numbers where the turbulent kinetic energy $K \sim t^{-5 / 2}-$ a power law consistent with the final period of decay. Batchelor (1948) also found a self-preserving solution to the Karman-Howarth equation in the limit of infinite Reynolds numbers for which Loitsianskii's integral was an invariant. This solution - wherein $L(t)$ is the integral length scale $\Lambda$ and $K \sim t^{-10 / 7}-$ was put forth by Batchelor as the only complete self-preserving solution at high Reynolds numbers. Of course, additional partial self-preserving solutions were shown by Batchelor to exist in other Reynolds number regimes.

Objections were later raised against the use of the Loitsianskii integral as a dynamic invariant: at high Reynolds numbers this integral can be shown to be a weak function of time (see Proudman and Reid 1954 and Batchelor and Proudman 1956). Saffman (1967) proposed an alternative dynamic invariant which yields a $K \sim t^{-6 / 5}$ power law decay in the infinite Reynolds number limit (see Hinze 1975). While the results of Batchelor and Saffman formally constitute complete self-preserving solutions to the inviscid KarmanHowarth equation, it must be kept in mind that they only exhibit partial self-preservation with respect to the full viscous equation. Namely, there is self-preservation only for the range of energy containing eddies with integral length scale $\Lambda$ (here, $\lambda / \Lambda<<1$ where $\lambda$ is the Taylor microscale). These two solutions have been widely accepted in the turbulence literature as the predicted decay laws for self-preserving isotropic turbulence at high Reynolds numbers.

Implicit in the analysis of Batchelor (1948) is the existence of a complete self-preserving solution consistent with high Reynolds numbers - namely a $K \sim t^{-1}$ power law decay. The collapsing length scale $L(t)$ for this full self-preserving solution is necessarily the Taylor microscale (i.e., for any complete self-preserving solution of the viscous Karman- 
Howarth equation we must have $L \propto \lambda)$. This solution - which was postulated earlier by Dryden (1943) - was dismissed by Batchelor on the grounds that Loitsianskii's integral was not a dynamic invariant. While this result by Dryden has been mentioned subsequently in the literature (c.f. Hinze 1975, Monin and Yaglom 1975 and Korneyev and Sedov 1976), it has largely been disregarded by the turbulence community. The reason for this appears to be two-fold: (a) a $K \sim t^{-1}$ power law decay has not been observed in the most accurate isotropic decay experiments, and (b) since $\lambda / \Lambda \rightarrow 0$ as $R e \rightarrow \infty$, doubts can be cast on the suitability of the Taylor microscale as the collapsing length scale of the energy containing eddies.

Recently, George $(1987,1989)$ revived this issue concerning the existence of complete self-preserving solutions in isotropic turbulence. In an interesting paper he claimed to find a complete self-preserving solution, valid for all Reynolds numbers, in which the kinetic energy decayed as $K \sim t^{-\alpha}$ with $\alpha$ determined by the initial conditions. George (1987) - who based his analysis on the dynamic equation for the energy spectrum rather than on the Karman-Howarth equation - made no explicit mention of the complete selfpreserving $K \sim t^{-1}$ solution. Strictly speaking, the solution presented by George was an alternative self-preserving solution to that of Karman and Howarth (1938) and Batchelor (1948) since he relaxed the constraint that the triple longitudinal velocity correlation be self-similar in the classical sense.

The purpose of the present paper is to address the issue of complete self-preservation in an effort to clarify the following basic questions:

(1) What is the complete self-preserving solution for isotropic turbulence at high Reynolds numbers,

(2) What detailed predictions does this solution yield for the energy decay, particularly during the initial approach to a state of complete self-preservation, and

(3) Is this solution compatible with the results of physical experiments and alternative theoretical approaches?

In so far as the first two points are concerned, it will be shown unequivocally that the only complete self-preserving solution that is consistent with a high-Reynolds-number isotropic turbulence has a $K \sim t^{-1}$ asymptotic power law decay. Unlike previous studies, this is demonstrated in a straightforward manner based on a fixed point analysis of the one-point equations. This analysis leads to an interesting interpretation of the physical significance of a $K \sim t^{-1}$ power law decay and allows us to examine small departures from a state of complete self-preservation. The detailed predictions of this complete self- 
preserving solution - which, to the best of our knowledge have never been examined in depth in the literature - will be compared with the results of physical experiments and alternative theoretical approaches in the sections to follow.

\section{THEORETICAL BACKGROUND}

We will consider isotropic turbulence governed by the incompressible Navier-Stokes equations

$$
\begin{gathered}
\frac{\partial u_{i}}{\partial t}+u_{j} \frac{\partial u_{i}}{\partial x_{j}}=-\frac{\partial p}{\partial x_{i}}+\nu \nabla^{2} u_{i} \\
\frac{\partial u_{i}}{\partial x_{i}}=0
\end{gathered}
$$

where $u_{i}$ is the velocity vector, $p$ is the pressure and $\nu$ is the kinematic viscosity. The two point double and triple longitudinal velocity correlations, denoted by $f(r, t)$ and $k(r, t)$, respectively, are defined in the standard way:

$$
\begin{aligned}
f(r, t) & =\frac{\overline{u(\mathbf{x}, t) u(\mathbf{x}+\mathbf{r}, t)}}{\overline{u^{2}}} \\
k(r, t) & =\frac{\overline{u^{2}(\mathbf{x}, t) u(\mathbf{x}+\mathbf{r}, t)}}{\left(\overline{u^{2}}\right)^{3 / 2}}
\end{aligned}
$$

where $u$ is any component of the velocity, $\mathbf{x}$ and $\mathbf{x}+\mathbf{r}$ are any two spatial points separated by a distance $r=|\mathbf{r}|$ in the direction of $u$, and an overbar denotes a spatial average (c.f. Hinze 1975). For isotropic turbulence, $f$ and $k$ satisfy the Karman-Howarth equation

$$
\frac{\partial\left(\overline{u^{2}} f\right)}{\partial t}=\left(\overline{u^{2}}\right)^{3 / 2}\left(\frac{\partial k}{\partial r}+\frac{4}{r} k\right)+2 \nu \overline{u^{2}}\left(\frac{\partial^{2} f}{\partial r^{2}}+\frac{4}{r} \frac{\partial f}{\partial r}\right)
$$

which is obtained directly from the Navier-Stokes equations. The turbulent kinetic energy $K \equiv \frac{1}{2} \overline{u_{i} u_{i}}$ is a solution of the differential equation

$$
\dot{K}=-\epsilon
$$

where

$$
\epsilon=\nu \overline{\omega_{i} \omega_{i}} \equiv \nu \omega^{2}
$$

is the turbulent dissipaton rate, $\omega_{i}$ is the vorticity vector and $\omega^{2}$ is the enstrophy. The turbulent dissipation rate is a solution of the differential equation

$$
\dot{\epsilon}=\frac{7}{3 \sqrt{15}} S_{K} \sqrt{R_{t}} \frac{\epsilon^{2}}{K}-\frac{7}{15} G \frac{\epsilon^{2}}{K}
$$


where

$$
\begin{gathered}
S_{K}=-\frac{\overline{\left(\frac{\partial u}{\partial x}\right)^{3}}}{\left[\sqrt{\left(\frac{\partial u}{\partial x}\right)^{2}}\right]^{\frac{3}{2}}}=-\lambda^{3}\left[\frac{\partial^{3} k}{\partial r^{3}}\right]_{r=0} \\
G=\lambda^{4}\left[\frac{\partial^{4} f}{\partial r^{4}}\right]_{r=0} \\
R_{t}=\frac{K^{2}}{\nu \epsilon}, \quad \lambda=\left(\frac{10 \nu K}{\epsilon}\right)^{1 / 2}
\end{gathered}
$$

are the velocity derivative skewness, the coefficient of the enstrophy destruction, the turbulence Reynolds number, and the Taylor microscale, respectively. Equations (6) and (8) - which are rearranged versions of those that appear in Karman and Howarth (1938) and Batchelor (1948) - are obtained, respectively, by setting $r=0$ in (5) and in the equation obtained by differentiating (5) twice with respect to $r$. Since $S_{K}=S_{K}(t)$ and $G=G(t)$ are directly related to the correlations $f$ and $k$ (which cannot both be obtained from the Karman-Howarth equation (5)) it is clear that the problem of isotropic decay is not closed. In order to achieve closure, additional assumptions must be made such as the one of complete self-preservation that will be discussed in this paper.

For an isotropic turbulence to be self-preserving in the sense of Karman and Howarth (1938) and Batchelor (1948), we must have

$$
\begin{aligned}
& f(r, t)=\tilde{f}(r / L) \\
& k(r, t)=\tilde{k}(r / L)
\end{aligned}
$$

where $L=L(t)$ is a uniquely specified similarity length scale. For it to exhibit complete self-preservation, all scales of the turbulence - namely, the full range of $0 \leq r \leq \infty$ - must decay according to (12) and (13) (partial self-preservation is satisfied if (12) (13) only apply to some restricted range of $0 \leq r \leq r_{\max }$ ). We will focus our attention on complete self-preserving solutions in the analysis to follow. In view of the identity (Batchelor 1948)

$$
\epsilon=-10 \nu K\left[\frac{\partial^{2} f}{\partial r^{2}}\right]_{r=0}
$$

it follows from (11) that

$$
\lambda^{2}\left[\frac{\partial^{2} f}{\partial r^{2}}\right]_{r=0}=-1
$$


Hence, for any complete self-preserving isotropic turbulence, we must have

$$
\frac{\lambda^{2}}{L^{2}} \tilde{f}^{\prime \prime}(0)=-1
$$

from which it can be concluded that

$$
L \propto \lambda
$$

since $\tilde{f}^{\prime \prime}(0)$ is a constant. It therefore follows that the Taylor microscale is the only similarity length scale that can yield complete self-preserving solutions to the full viscous equations of motion for isotropic turbulence.

Without any loss of generality for a complete self-preserving isotropic turbulence we may set $L=\lambda$ and then substitute (12) - (13) into (9) - (10), respectively, to get

$$
\begin{gathered}
S_{K}=-\tilde{k}^{\prime \prime \prime}(0)=\mathrm{constant} \\
G=\tilde{f}^{i v}(0)=\mathrm{constant}
\end{gathered}
$$

where a prime denotes a derivative with respect to $\eta \equiv r / \lambda$. Consequently,

$$
S_{K}=S_{K_{o}}, \quad G=G_{o}
$$

where the notation $(\cdot)_{o}$ denotes the initial value. The substitution of (18) into (6) and (8) then yields the transport equations

$$
\begin{gathered}
\dot{K}=-\epsilon \\
\dot{\epsilon}=\frac{7}{3 \sqrt{15}} S_{K_{o}} \sqrt{R_{t}} \frac{\epsilon^{2}}{K}-\frac{7}{15} G_{o} \frac{\epsilon^{2}}{K}
\end{gathered}
$$

for complete self-preserving isotropic turbulence. This is a closed system for the determination of $K$ and $\epsilon$ once $K_{o}, \epsilon_{o}, S_{K_{o}}$ and $G_{o}$ are provided. To simplify the subsequent presentation, the quantity $G_{0}$ - which is the coefficient of the destruction of enstrophy term in (20) - will henceforth be referred to as the "palinstrophy coefficient" following the terminology used by Lesieur (1990). Accordingly, the assumption of complete selfpreservation is seen to lead to closure in the following sense: if initial conditions for the skewness and the palinstrophy coefficient are provided - in addition to initial conditions for $K$ and $\epsilon$ - then the energy decay can be calculated explicitly for all later times.

For complete self-preserving isotropic turbulence, the Karman-Howarth equation (5) takes the form

$$
\dot{K} \tilde{f}-K \frac{\dot{\lambda}}{\lambda} \eta \frac{d \tilde{f}}{d \eta}-\sqrt{\frac{2}{3}} \frac{K}{\lambda} \eta^{-4} \frac{d\left(\eta^{4} \tilde{k}\right)}{d \eta}-2 \nu \frac{K}{\lambda^{2}} \eta^{-4} \frac{d}{d \eta}\left(\eta^{4} \frac{d \tilde{f}}{d \eta}\right)=0
$$


or, equivalently,

$$
10 \tilde{f}+2 \eta^{-4} \frac{d}{d \eta}\left(\eta^{4} \frac{d \tilde{f}}{d \eta}\right)+\eta \frac{d \tilde{f}}{d \eta}\left(\frac{7}{3} G_{\circ}-5\right)=R_{\lambda}\left(\frac{7}{6} S_{K_{\circ}} \eta \frac{d \tilde{f}}{d \eta}-\eta^{-4} \frac{d\left(\eta^{4} \tilde{k}\right)}{d \eta}\right)
$$

after replacing $\dot{\lambda}$ using (11), (19) and (20) with $R_{\lambda}=\left(\overline{u^{2}}\right)^{1 / 2} \lambda / \nu=\sqrt{20 / 3} R_{t}^{1 / 2}$. Equation (22) will have a solution if $R_{\lambda}=$ constant as first noticed by Dryden (1943); this is a $K \sim t^{-1}$ power law decay. However, (22) also has solutions where $R_{\lambda}=R_{\lambda}(t)$ when separability is invoked. The separability condition implies that each side of (22) is equal to zero individually, yielding differential equations from which explicit solutions for $\tilde{f}$ and $\tilde{k}$ may be determined depending on the choice of $S_{K_{0}}$ and $G_{0}$. These solutions were first discovered by Sedov (1944) and later compared with experimental data by Korneyev and Sedov (1976). The particular case for which $G_{o}=3$ so that $\tilde{f}$ is Gaussian - which formally corresponds to the final period of decay - was considered in detail by Bernard (1985). We will briefly examine (22) later to establish the consistency of the present results with those of previous studies. However, our analyses will be based on a fixed point analysis and direct numerical integration of (19) - (20). This will allow us to consider small departures from a self-preserving state as will be demonstrated later.

\section{FIXED POINT ANALYSIS AND NUMERICAL RESULTS}

In order to carry out a fixed point analysis of (19) and (20), we will combine these equations into a single transport equation for the turbulence Reynolds number $R_{t}$. Since,

$$
\dot{R}_{t}=\frac{2 K \dot{K}}{\nu \epsilon}-\frac{K^{2}}{\nu \epsilon^{2}} \dot{\epsilon}
$$

it follows that

$$
\dot{R}_{t}=-\frac{2 K}{\nu}-\frac{7}{3 \sqrt{15}} S_{K_{\circ}} \sqrt{R_{t}} \frac{K}{\nu}+\frac{7}{15} G_{o} \frac{K}{\nu} .
$$

If the transformed dimensionless time $\tau$ - defined by the relation $d \tau=(\epsilon / K) d t-$ is introduced into (24), we obtain the equation

$$
\frac{d R_{t}}{d \tau}=R_{t}\left(\frac{7}{15} G_{o}-2-\frac{7}{3 \sqrt{15}} S_{K_{o}} \sqrt{R_{t}}\right)
$$

The fixed points of (25) are obtained by setting $d R_{t} / d \tau=0$ which yields the equation:

$$
R_{t_{\infty}}\left(\frac{7}{15} G_{o}-2-\frac{7}{3 \sqrt{15}} S_{K_{\circ}} \sqrt{R_{t_{\infty}}}\right)=0
$$


where $(\cdot)_{\infty}$ denotes the equilibrium value in the limit as $\tau \rightarrow \infty$. Equation (26) has the solutions:

$$
R_{t_{\infty}}=0
$$

for $\frac{7}{15} G_{\circ} \leq 2$, and

$$
R_{t_{\infty}}=\left(\frac{\frac{7}{15} G_{o}-2}{\frac{7}{3 \sqrt{15}} S_{K_{o}}}\right)^{2}
$$

for $\frac{7}{15} G_{0}>2$. It is a simple matter to show that the fixed points (27) - (28) are stable nodes that attract all initial conditions $K_{o}$ and $\epsilon_{o}$. It is also evident from (28) that in order to have an equilibrium high-Reynolds-number isotropic flow field ${ }^{\dagger}$ under self-preserving conditions it is necessary that $G_{0} \sim \sqrt{R_{t_{\infty}}}$.

The fixed point $R_{t_{\infty}}=0$ is associated with asymptotic solutions of $K$ and $\epsilon$ that satisfy the differential equations

$$
\begin{gathered}
\dot{K}=-\epsilon \\
\dot{\epsilon}=-\frac{7}{15} G_{\circ} \frac{\epsilon^{2}}{K} .
\end{gathered}
$$

Equations (29) - (30) have the exact solution

$$
\begin{aligned}
& K=K_{\circ}\left(1+\frac{1}{\alpha} \frac{\epsilon_{o} t}{K_{o}}\right)^{-\alpha} \\
& \epsilon=\epsilon_{\circ}\left(1+\frac{1}{\alpha} \frac{\epsilon_{o} t}{K_{o}}\right)^{-\alpha-1}
\end{aligned}
$$

where $\alpha=1 /\left(\frac{7}{15} G_{o}-1\right)>1$. For large eddy turnover times $\epsilon_{0} t / K_{0} \gg 1$, we have

$$
\begin{gathered}
K \sim t^{-\alpha} \\
R_{t} \sim t^{-\alpha+1}
\end{gathered}
$$

(and, hence, $K$ goes to zero faster than $R_{t}$ ), so it follows that (31) - (32) can only be asymptotic solutions in the limit as $K \rightarrow 0$ and $t \rightarrow \infty$. Therefore, within the framework of complete self-preservation, an asymptotic power law decay where $K \sim t^{-\alpha}$ (with $\alpha>1$ ) is associated with the fixed point $R_{t_{\infty}}=0$ and is only formally consistent with the final period of decay. This is borne out in the subsequent computations.

During the final period of decay there is considerable evidence (Batchelor and Townsend 1948a) indicating that $\tilde{f}(\eta)=\exp \left(-\eta^{2} / 2\right)$ (i.e., a Gaussian) in which case (10) implies

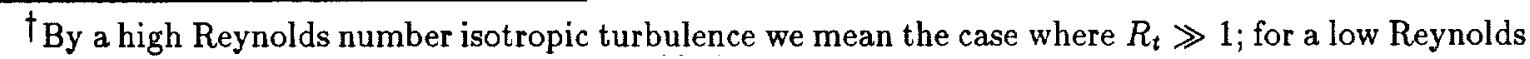
number isotropic turbulence, $R_{t}=O(1)$. 
that $G_{o}=3$ and, consequently, that $\alpha=5 / 2$. The same result is also reached by assuming constancy of the Loitsianskii integral

$$
\overline{u^{2}} \int_{0}^{\infty} r^{4} f(r, t) d r=\text { constant }
$$

during decay, which appears to be an acceptable assumption for the final period. In particular, from (12) it follows that (35) is equivalent to (c.f. Hinze 1975)

$$
\overline{u^{2}} \lambda^{5} \int_{0}^{\infty} \eta^{4} \tilde{f}(\eta) d \eta=\text { constant }
$$

so that (11) and (36) imply that

$$
\frac{K^{7 / 2}}{\epsilon^{5 / 2}}=\text { constant }
$$

When (37) is combined with (31) and (32) it follows that $\alpha=5 / 2$ - the celebrated Batchelor (1948) result.

Now we will show that the non-zero fixed point (28) is consistent with high-Reynoldsnumber isotropic turbulence. The substitution of (28) into (19) - (20) yields the equations

$$
\begin{gathered}
\dot{K}=-\epsilon \\
\dot{\epsilon}=-2 \frac{\epsilon^{2}}{K}
\end{gathered}
$$

which have the exact solution

$$
\begin{gathered}
K=K_{o}\left(1+\frac{\epsilon_{o} t}{K_{o}}\right)^{-1} \\
\epsilon=\epsilon_{o}\left(1+\frac{\epsilon_{o} t}{K_{o}}\right)^{-2}
\end{gathered}
$$

(namely, a $t^{-1}$ power law decay for the turbulent kinetic energy). Since it can be shown that (see Batchelor and Townsend 1948b)

$$
G=\frac{30 \nu}{7} \frac{\frac{\overline{\partial \omega_{j}}}{\partial x_{j} \frac{\partial \omega_{i}}{\partial x_{j}}} / \overline{\omega_{k} \omega_{k}}}{\epsilon / K}
$$

it follows that $G$ is a ratio of turbulent to dissipative time scales. It is a simple matter to show that (see Hinze 1975)

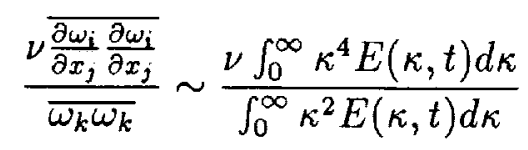


where $E(\kappa, t)$ is the energy spectrum and $\kappa$ is the wavenumber. For complete selfpreservation, $E(\kappa, t)$ scales with the Taylor microscale, from which it follows that

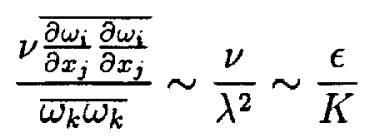

and, hence, that

$$
G \sim \text { constant }
$$

as shown earlier. However, (43) is a correlation dominated by the high wavenumbers (i.e., small scales) and it would therefore seem more reasonable that $E(\kappa, t)$ should scale with the Kolmogorov length scale $l_{K} \equiv \nu^{3 / 4} / \epsilon^{1 / 4}$. If this is the case, then

$$
\frac{\nu \overline{\frac{\partial \omega_{i} \frac{\partial \omega_{i}}{\partial x_{j}}}{\partial x_{j}}}}{\overline{\omega_{k} \omega_{k}}} \sim \frac{\nu}{l_{K}^{2}}
$$

and

$$
G \sim \frac{\nu}{l_{K}^{2}} \frac{K}{\epsilon} \sim \sqrt{R_{t}} .
$$

Equations (45) and (47) appear to be contradictory; however, they are not, since in a $K \sim t^{-1}$ power law decay

$$
R_{t}=\text { constant }
$$

Furthermore, $R_{t}=$ constant resolves the imbalance between the two terms on the righthand side of the dissipation rate equation (20) since the first term (i.e., the vortex stretching) is initially of $O\left(\sqrt{R_{t}}\right)$ while the second term (i.e., the viscous destruction) is of $O(1)$. This leads us to the following physical interpretation: $A K \sim t^{-1}$ power law decay is the asymptotic state toward which a self-preserving isotropic turbulence is driven at high Reynolds numbers in order to resolve the fundamental imbalance between vortex stretching and viscous diffusion. In the process of resolving this imbalance, compatibility with Kolmogorov scaling is achieved for the small scale correlations. Since this consistency with Kolmogorov scaling - which, on physical grounds should be satisfied at high Reynolds numbers - is achieved when $\frac{7}{15} G_{o} \sim \sqrt{R_{t_{\infty}}}>2$, it is clear that the non-zero fixed point (28) is the asymptotic solution for high-Reynolds-number self-preserving flows.

We will now examine numerical solutions of (19) - (20) for complete self-preservation. With the possible exception of the recent work of Bernard (1985), we have not seen detailed numerical results published on the decay of $K$ and $\epsilon$ in self-preserving isotropic turbulence. An examination of these results will amplify the points discussed in this 
section and will shed some interesting new light on how the self-preservation assumption compares with experiments. In Figures 1(a) - (c), the decay of the turbulent kinetic energy is shown for three initial turbulence Reynolds numbers $\left(R_{t_{o}}=1000,5000\right.$ and $10,000)$ and three different initial conditions for $G$ (i.e., $C_{\epsilon_{2}}=1.92,5.0$, and 8.0 where $C_{\epsilon_{2}}=\frac{7}{15} G_{o}$ ). For these calculations, as well as the ones to follow, $K^{*} \equiv K / K_{o}, t^{*} \equiv$ $\epsilon_{0} t / K_{0}$ and the skewness

$$
S_{K_{o}}=0.5
$$

which is in close proximity to the values obtained from physical experiments in this range of Reynolds numbers. From these figures it is clear that the self-preserving solution has an initial transient where the kinetic energy is fairly flat; then the kinetic energy begins to asymptote from above to a power law decay as evidenced by a straight line on these logarithmic plots. Two conclusions can be drawn from these results. First, for $C_{\epsilon_{2}}<2$ and $R_{t_{o}} \gg 1$, the kinetic energy does not asymptote to a $t^{-\alpha}$ power law decay until after an extremely large number of eddy turnover times by which time the turbulence has decayed to a tiny fraction of its initial intensity. Second, for $C_{\epsilon_{2}}>2$, the kinetic energy asymptotes to a $t^{-1}$ power law decay within a few eddy turnover times; however unless $C_{\epsilon_{2}} \sim \sqrt{R_{t_{o}}}$ (consistent with Kolmogorov scaling), the turbulence intensity will drop precipitously before this asymptotic state is achieved.

To further illustrate these points, the computed turbulent kinetic energy is compared with its corresponding asymptotic power law decay for increasing values of $C_{\epsilon_{2}}$ in Figures 2(a) - (c) which are for initial turbulence Reynolds numbers of 1000, 10,000 and 100,000, respectively. It is clear from these figures that for $C_{\epsilon_{2}}<2$, the turbulent kinetic energy does not reach its asymptotic power law decay even after 100 eddy turnover times! However, for $C_{\epsilon_{2}}$ sufficiently larger than 2 , the turbulent kinetic energy asymptotes to a $t^{-1}$ power law decay within a few eddy turnover times. This can be seen even more vividly in Figure 3 where $\alpha^{*} \equiv d\left(\log K^{*}\right) / d\left(\log t^{*}\right)$ is plotted as a function of $\log t^{*}$ for $R_{t_{o}}=1000$. If there is an asymptotic power law decay, this derivative will asymptote to the exponent of the decay law. It is clear that for $C_{\epsilon_{2}}=7$, an exponent of 1 is approached quickly; however, for $C_{t_{2}}=1.83$ and 1.92 (initial conditions which ultimately yield a power law decay with an exponent of approximately 1.2 and 1.1, respectively) an asymptotic state is not achieved even after 100 eddy turnover times. Furthermore, for $C_{\epsilon_{2}}<2$ and large initial turbulence Reynolds numbers $R_{t_{0}} \gg 1$, there is a precipitous drop in the turbulent kinetic energy before a power law decay is achieved; this is due to the early transient when vortex stretching causes a considerable rise in the dissipation (see Figures 4 (a) - 
(b)).

Since the self-preserving solutions for $C_{\epsilon_{2}}<2$ only asymptote to a power law decay in the limit as $K \rightarrow 0$ and $t \rightarrow \infty$, it is reasonable to associate them exclusively with the final period of decay. Experiments tend to indicate that the final period of decay is entered for $R_{t}<1$ wherein the exponent of the decay is approximately 2.5 (c.f. Hinze 1975). As noted earlier, this decay law is obtained asymptotically for self-preserving isotropic turbulence if $C_{\epsilon_{2}}=1.4$ - a result obtained by invoking Loitsianskii's invariant. In Figure 5, the decay of the turbulent kinetic energy when $C_{\epsilon_{2}}=1.4$ is shown for the initial turbulence Reynolds numbers $R_{t_{0}}=0.25$ and 1.0. It is clear from these results that for $R_{t_{\mathrm{o}}}<1$ the solutions begin to asymptote to the power law decay (31) with an exponent $\alpha=2.5$. Since $C_{\epsilon_{2}}$ must equal 1.4 during the final period of decay - and, since $C_{\epsilon_{2}}$ is a constant for self-preserving isotropic turbulence - it follows that the entire decay process from high Reynolds number initial conditions to the final period of decay cannot be described within the framework of complete self-preservation. This conclusion results from the fact that the only consistent self-preserving solution at high initial Reynolds numbers yields a $K \sim t^{-1}$ power law decay wherein $R_{t}$ asymptotes to a constant - a state of affairs that precludes the description of the later stages of decay. Furthermore, the value of $C_{\epsilon_{2}}=1.4$, which describes the final period of decay, yields unphysical results for the early stages of a high Reynolds number isotropic turbulence (i.e., it predicts an early time transient where there is a precipitous drop in the turbulent kinetic energy; see Figure 6). In order to describe the entire decay process of a high Reynolds number isotropic turbulence, $G$ as well as $S_{K}$ must vary with time - a possibility that is precluded by the assumption of self-preservation which renders them to be constant.

\section{COMPARISONS WITH ALTERNATIVE THEORETICAL ANALYSES AND EXPERIMENTS}

The results derived in the previous section are consistent with those of Batchelor (1948) for low turbulence Reynolds numbers; however, our high Reynolds number asymptotic solution yields $K \sim t^{-1}$ whereas in Batchelor's solution $K \sim t^{-10 / 7}$. The reason for this difference is simple: as alluded to earlier, Batchelor also found the $K \sim t^{-1}$ solution but dismissed it as a viable result since Loitsianskii's integral was not a dynamic invariant. Interestingly enough, an earlier experimental study by Batchelor and Townsend (1948b) yielded results that were far more suggestive of a $K \sim t^{-1}$ rather 
than a $K \sim t^{-10 / 7}$ power law decay. Despite the fact that Batchelor (1948) states that the $K \sim t^{-10 / 7}$ decay law is a complete self-preserving solution, in reality it is only a partial self-preserving solution corresponding to the inviscid Karman-Howarth equation (see Hinze 1975). It is our view that since the $K \sim t^{-1}$ asymptotic decay law is a formal solution to the full Karman-Howarth equation, it should not be dismissed unless it is in incontrovertible contradiction of experiments or other exact theoretical results.

As mentioned earlier, Dryden (1943) postulated a $K \sim t^{-1}$ power law decay based on a direct analysis of the Karman-Howarth equation. He observed - as is evident from (22) - that the Karman-Howarth equation will allow for self-similar solutions if

$$
R_{\lambda}=\text { constant }
$$

which yields $K \sim t^{-1}$ as a direct consequence of (6). However, there are other temporally varying solutions to (22); complete self-preservation only requires that $R_{\lambda}$ asymptote to a constant. Sedov (1944) studied solutions of (22) obtained by applying the separability constraint

$$
\begin{gathered}
10 \tilde{f}+2 \eta^{-4} \frac{d}{d \eta}\left(\eta^{4} \frac{d \tilde{f}}{d \eta}\right)+\eta \frac{d \tilde{f}}{d \eta}\left(\frac{7}{3} G_{o}-5\right)=0 \\
\left(\frac{7}{6} S_{K_{\circ}} \eta \frac{d \tilde{f}}{d \eta}-\eta^{-4} \frac{d\left(\eta^{4} \tilde{k}\right)}{d \eta}\right)=0
\end{gathered}
$$

which renders $R_{\lambda}=R_{\lambda}(t)$ consistent with (19) - (20). Solutions to (51) - (52) have not been studied in great depth subsequent to Sedov (1944) who showed that $\tilde{f}(\eta)=$ $M\left(\frac{5}{2 \gamma}, \frac{5}{2},-\frac{\gamma \eta^{2}}{2}\right)$ where $M$ is the confluent hypergeometric function and $\gamma=\frac{7}{6} G_{o}-\frac{5}{2}$. Batchelor (1948) expressed concern over the fact that this solution leads to a unique determination of both $\tilde{f}$ and $\tilde{k}$; however, although he suspected that the Sedov solution was unphysical, he stated that he was "not able to find any definite anomalies". In the limit as $R_{\lambda} \rightarrow 0$ it is clear that (51) is a direct consequence of the Karman-Howarth equation. Consequently, it is not surprising that the Sedov solution for the final period of decay yields physically interesting solutions as recently demonstrated by Bernard (1985). However, it will now be shown definitively that the Sedov solution yields unphysical results at high turbulence Reynolds numbers. In figure 7 , the results of a numerical solution of (51) for $\tilde{f}$ are shown for a variety of values of $G_{0}$ ranging from 3 to 60 . For $G_{o}=3$ it can be shown analytically that $\tilde{f}=\exp \left(-\eta^{2} / 2\right)$ yielding an energy spectrum of the form

$$
E^{*}\left(\kappa^{*}\right)=\frac{1}{\sqrt{2 \pi}} \kappa^{*^{4}} \exp \left(-\kappa^{*^{2}} / 2\right)
$$


where $\kappa^{*}=\kappa \lambda$ and $E^{*}=E / \overline{u^{2}} \lambda$. Equation (53) is obtained from the identity

$$
E^{*}\left(\kappa^{*}\right)=\frac{1}{\pi} \int_{0}^{\infty} \tilde{f}(\eta)\left(\kappa^{*} \eta \sin \kappa^{*} \eta-\kappa^{*^{2}} \eta^{2} \cos \kappa^{*} \eta\right) d \eta
$$

(c.f. Batchelor 1953). This result - which has $E^{*}\left(\kappa^{*}\right) \sim \kappa^{*}$ at low wavenumbers and has $E^{*}\left(\kappa^{*}\right)$ decaying exponentially at high wavenumbers - is consistent with established results on the final period of decay (c.f. Hinze 1975). However, for sufficiently large $G_{o}$, it is a simple matter to show from (51) that

$$
\tilde{f}(\eta) \sim \eta^{-\frac{5}{\gamma}}
$$

for $\eta \gg 1$. This explains why $\tilde{f}(\eta)$ is so slow to asymptote to zero when $G_{0}>10$ in figure 7 . In fact for

$$
\frac{7}{15} G_{0}>2
$$

it follows from (54) and (55) that $E^{*}\left(\kappa^{*}\right)$ becomes singular. From (28) it can then be concluded that the Sedov solution will yield a singular energy spectrum when

$$
R_{t_{\infty}}>0
$$

It is thus clear that the Sedov solution yields untenable results, at high Reynolds numbers, for the double and triple two-point longitudinal velocity correlations.

The major deficiency with the approaches of Dryden and Batchelor - as well as that of Sedov - lies in the use of the self-similar Karman-Howarth equation (22) which does not allow for the treatment of small departures from a state of complete self-preservation. Such small departures can be characterized by the perturbations

$$
\begin{gathered}
S_{K}=S_{K_{0}}+\delta S_{K}(t) \\
G=G_{\circ}+\delta G(t)
\end{gathered}
$$

where $\left\|\delta S_{K}\right\| / S_{K_{\circ}} \ll 1,\|\delta G\| / G_{\circ} \ll 1$ and $\delta S_{K}(t), \delta G(t) \rightarrow 0$ as $t \rightarrow \infty$. The substitution of (56) - (57) into (6) and (8) yields the governing equations for small departures from a state of self-preservation. If we denote $\delta K$ and $\delta \epsilon$ as the departures from the self-preserving solutions $K$ and $\epsilon$ obtained from (19) - (20), it follows that for the perturbations $(56)-(57)$ we will have

$$
\frac{\|\delta K\|}{\|K\|} \ll 1, \quad \frac{\|\delta \epsilon\|}{\|\epsilon\|} \ll 1
$$


due to the fact that (19) - (20) have fixed points that are stable nodes (c.f. Guckenheimer and Holmes 1986). Consequently, (19) - (20) will yield an excellent approximation for isotropic decay when there are extremely small departures from a state of complete selfpreservation. In contrast to this nice behavior, the Karman-Howarth equation becomes indeterminate when subjected to infinitesimal perturbations from a self-preserving state. Hence, it appears that the one-point equations (19) - (20) form a broader basis for the analysis of the energy decay of self-preserving isotropic decay than does (22).

The general solution to the complete self-preserving isotropic decay equations (19) (20) at high Reynolds numbers is shown schematically in figure 8 (this is for the physically significant case where $R_{t_{o}}>R_{t_{\infty}}$ so that the turbulence Reynolds number decays). There is an early time transient (region $A B$ ) where the turbulent kinetic energy is flat; it is eventually followed by the asymptotic region $C D$ where $K \sim t^{-1}$. These two regions are connected by the overlap region $B C$. The initial transient $A B$ evolves on the Kolmogorov time scale $\sqrt{\nu / \epsilon}$ during which time there is a precipitous drop in the turbulence Reynolds number (see figure 9). On the other hand, the overlap region $B C$ evolves on the turbulence time scale $K / \epsilon$; in this region the turbulence Reynolds number $R_{t}$ becomes close to $R_{t_{\infty}}$, approaching it asymptotically from above. As a direct consequence of the perturbation analysis discussed above, the overlap region $B C$ can be set into strong approximate agreement with the asymptotic approach to a state of complete self-preservation. These results have a direct bearing on how the complete self-preserving solution compares with physical experiments as we will soon see.

It is widely believed that a $K \sim t^{-1}$ asymptotic decay law is in violation of experimental data for isotropic turbulence. This experimental data (see Uberoi 1963, Kistler and Vrebalovich 1966, Comte-Bellot and Corrsin 1966, 1971, and Warhaft and Lumley 1978) has yielded power law decays with exponents varying from 1 to 1.4 with a mean of approximately 1.25 . However, great caution must be taken in using this data to dismiss the possibility of a $K \sim t^{-1}$ asymptotic power law decay at high Reynolds numbers since most of this data is for a limited number of eddy turnover times (typically for $\epsilon_{o} t / K_{0}<4$ ). If the self-preserving solution is examined for this same limited number of eddy turnover times it follows that the resulting solution can be fitted to an excellent degree of approximation by a power law decay with exponents in the range of 1 to 1.4 depending on the initial conditions; the lower the Reynolds number, the longer the solution takes to reach an asymptotic state and the larger the exponent is during the early stages of decay (see Figures $10(\mathrm{a})-(\mathrm{c}))$. Consequently, if one examined in isolation the self-preserving solu- 
tions for the first few eddy turnover times (with the short early time transient omitted), one could erroneously conclude that there was an asymptotic power law decay with an exponent in the range of 1 to 1.4 depending on the initial conditions; in reality, all of these solutions are asymptoting to a $t^{-1}$ power law decay. The solutions shown in Figures 10 (a) - (c) correspond to the overlap region $B C$ shown in Figure 8 and, hence, can be associated with the asymptotic approach to a state of complete self-preservation. An argument has been raised recently by Walker and Corrsin (1985) and Walker (1986) that the physical experiments may not go far enough to see a $t^{-1}$ power law decay. Unless the initial turbulence Reynolds number is extremely large, an asymptotic state may not be achieved in the first few eddy turnover times. In this regard it is interesting to note that the only extremely high-Reynolds-number experiment (i.e., Kistler and Vrebalovich 1966) and large-eddy turnover time experiment (Walker 1986) did measure a $K \sim t^{-1}$ asymptotic power law decay. Consequently, existing experiments cannot rule out the possibility of a $K \sim t^{-1}$ asymptotic power law decay at high Reynolds numbers and do not warrant the dismissal of the complete self-preserving solution discussed herein. Furthermore, Rosen (1981) recently derived a $t^{-1}$ asymptotic power law decay based on alternative ideas from statistical mechanics.

Now, we will address the interesting controversy generated recently by George (1987, 1989). He claimed to find complete self-preserving solutions - with the Taylor microscale as the similarity length scale - that exist for all turbulence Reynolds numbers. These solutions were characterized by an asymptotic power law decay where the exponent is determined by the initial conditions. George arrived at this alternative self-preserving solution by relaxing the classical similarity constraint (13). He argued that the normalization of the two-point triple velocity correlation

$$
T(r, t)=\overline{u^{2}(\mathbf{x}, t) u(\mathbf{x}+\mathbf{r}, t)}
$$

by $\left(\overline{u^{2}}\right)^{3 / 2}$ to form $k(r, t)$ is arbitrary since its one-point contraction $T(0, t) \equiv \overline{u^{3}}$ is zero. (This stands in contrast to the formulation of $f(r, t)$ which is obtained by normalizing the two-point double velocity correlation with its one-point contraction $\overline{u^{2}}$ ). Consequently, George argued that constraint (13) should be replaced with the alternative constraint

$$
\frac{T(r, t)}{w(t)}=\tilde{T}\left(\frac{r}{\lambda}\right)
$$

where $w(t)$ is a suitable weighting function. Then - from the definition of $S_{K}$ in equation 
(9) and the Karman-Howarth equation (22) - instead of the constraints

$$
R_{\lambda}=\text { constant }, \quad S_{K}=\text { constant }
$$

which renders a $t^{-1}$ power law decay, we get the constraints

$$
R_{\lambda} S_{K}=\text { constant }, \quad R_{\lambda} w K^{-3 / 2}=\text { constant }
$$

which allows for the possibility of an alternative decay law within the general framework of complete self-preservation. From (61) it follows that

$$
S_{K} \propto R_{t}^{-1 / 2}
$$

and that

$$
w \propto K^{3 / 2} R_{t}^{-1 / 2},
$$

which shows, incidentally, that $w$ cannot be chosen arbitrarily. Since the proportionality constant in (62) must be $S_{K_{o}} R_{t_{o}}^{1 / 2}$ - and since $G$ still remains a constant $G_{o}$ during the decay - this alternative self-preservation leads to the decay equations

$$
\begin{gathered}
\dot{K}=-\epsilon \\
\dot{\epsilon}=\frac{7}{3 \sqrt{15}} S_{K_{o}} R_{t_{o}}^{1 / 2} \frac{\epsilon^{2}}{K}-\frac{7}{15} G_{o} \frac{\epsilon^{2}}{K}
\end{gathered}
$$

instead of (19) - (20). Equations (64) - (65) yield the closed form solution for the energy decay

$$
K=K_{o}\left(1+\frac{1}{\beta} \frac{\epsilon_{o} t}{K_{o}}\right)^{-\beta}
$$

where

$$
\beta=\left(\frac{7}{15} G_{0}-\frac{7}{3 \sqrt{15}} S_{K_{0}} R_{t_{o}}^{1 / 2}-1\right)^{-1} .
$$

This is indeed a power law decay with an exponent that depends on the initial conditions as claimed by George $(1987,1989)$.

Although we do feel that George raised some interesting issues - and made a seemingly valid point concerning the arbitrariness of the normalization of the two-point triple velocity correlation - it is our opinion that there is a problem with this alternative self-preserving solution. By virtue of (61), George's alternative scaling renders a zero skewness in the limit of infinite turbulence Reynolds numbers. Experiments indicate 
that the skewness is an absolute constant of order one at extremely high Reynolds numbers (see Van Atta and Antonia 1980). Furthermore, a direct physical consequence of a vanishing skewness $S_{K}$ is the occurrence of zero transfer and, hence, no energy cascade. This can be seen easily from equation (59) which can be re-written as

$$
T(r, t) \propto K^{3 / 2} R_{t}^{-1 / 2} \tilde{T}\left(\frac{r}{\lambda}\right)
$$

which implies that the transfer $T(r, t) \rightarrow 0$ as $R_{t} \rightarrow \infty$. Vanishing transfer in the limit of infinite Reynolds numbers is an untenable physical result and hence this alternative self-preserving solution is not acceptable for high-Reynolds-number isotropic turbulence.

Finally, a few comments are in order concerning the implications of these results for turbulence modeling. In the commonly used turbulence models, the dissipation rate equation is modeled as

$$
\dot{\epsilon}=-C_{\epsilon_{2}} \frac{\epsilon^{2}}{K}
$$

for isotropic decay, where $C_{\epsilon_{2}}$ is a constant (c.f. Launder and Spalding 1974, Speziale 1991). Equation (68) is derived by invoking Kolmogorov scaling for $G$ which requires that

$$
G=C_{1} \sqrt{R_{t}}+C_{2}
$$

where $C_{1}$ and $C_{2}$ are constants; an equilibrium hypothesis is then made by which $C_{1}=$ $\frac{\sqrt{15}}{3} S_{K}$ so that the leading order part of the destruction of dissipation term annihilates the vortex stretching term in (8) yielding (68). In contrast to (68), the complete selfpreserving solution has a dissipation rate transport equation of the general mathematical form

$$
\dot{\epsilon}=C_{\epsilon_{3}} \sqrt{R_{t}} \frac{\epsilon^{2}}{K}-C_{\epsilon_{2}} \frac{\epsilon^{2}}{K}
$$

where $C_{\epsilon_{2}}$ and $C_{\epsilon_{3}}$ are constants. Equation (70) can be also derived based on Kolmogorov scaling (69) when departures from equilibrium are allowed wherein $C_{1} \neq \frac{\sqrt{15}}{3} S_{K}$. The addition of the unbalanced vortex stretching term in (70) allows for a better treatment of departures from equilibrium in several ways. First, as shown earlier in Figures 10 (a) (c), the self-preservation model allows for the description of the initial stages of isotropic decay where the exponent of the decay law can vary mildly with the initial conditions a feature observed in physical experiments. On the other hand, the more commonly used model (68) predicts a universal decay law where $K \sim t^{-\frac{1}{\epsilon_{\epsilon_{2}}-1}}$ for all $R_{t_{\circ}}$ and all $t>0$. Second, the self-preserving solution can accommodate the limit of zero viscosity. In this 
limit, it is a simple matter to show that (20) predicts a finite time enstrophy blow-up at the critical time

$$
t_{c}=\frac{6 \sqrt{15}}{7 S_{K_{o}} \omega_{o}}
$$

where $\omega_{o}^{2}$ is the initial enstrophy. In Figure 11(a), the time evolution of the enstrophy corresponding to the complete self-preserving solution is shown for a variety of increasing $R_{t_{o}}$; it is clear that an enstrophy crisis is predicted for $R_{t_{o}} \gg 1$ which eventually leads to a finite time enstrophy blow-up in the limit as $\nu \rightarrow 0$. These results are in excellent qualitative agreement with results obtained from EDQNM as illustrated in Figure 11(b) taken from Lesieur (1990). While the issue of a finite time enstrophy blow-up is still being debated by the turbulence community (c.f. Pumir and Siggia 1990), one thing is clear: the enstrophy grows dramatically when $\nu=0$. In contrast to the results shown in Figures 11 (a) - (b), the commonly used dissipation rate model (68) erroneously predicts that the enstrophy is conserved in the inviscid limit, i.e. that

$$
\omega^{2}=\text { constant }
$$

when $\nu=0$. It thus appears that the complete self-preserving solution allows for a better treatment of non-equilibrium isotropic turbulence that could be of future use in the development of improved turbulence models.

\section{CONCLUSIONS}

The energy decay for complete self-preserving isotropic turbulence has been re-examined from a basic theoretical and computational standpoint. Several interesting conclusions can be drawn from these results:

(1) The nonlinear differential equations for the energy decay have two fixed points $R_{t_{\infty}}=0$ and $R_{t_{\infty}}=\frac{135}{49}\left(\frac{7}{15} G_{o}-2\right)^{2} / S_{K_{o}}^{2}$. The former fixed point is only achieved in the limit as $t \rightarrow \infty$ and hence is associated with the final period of decay. Consistent with the Batchelor (1948) result, a $K \sim t^{-5 / 2}$ power law decay is obtained when Loitsianskii's invariant or the Gaussianity of $\tilde{f}(\eta)$ is invoked.

(2) The non-zero fixed point $R_{t_{\infty}}=\frac{135}{49}\left(\frac{7}{15} G_{o}-2\right)^{2} / S_{K_{o}}^{2}$ is approached within a few eddy turnover times and gives rise to a $K \sim t^{-1}$ asymptotic power law decay. It is the high-Reynolds-number asymptotic solution for a complete self-preserving isotropic turbulence. This solution appears to have been prematurely dismissed by Batchelor (1948) 
purely on the grounds that Loitsianskii's integral was not an invariant - a constraint which was later learned to be violated in isotropic turbulence when $R_{t_{\circ}} \gg 1$.

(3) The structure of the high Reynolds-number self-preserving solution during the first few eddy turnover times was examined in detail. By a perturbation analysis, it was argued that these solutions can serve as an approximation for the asymptotic approach to a state of complete self-preservation. It was found that, depending on the initial conditions, the early time solutions could be fitted with a power law decay which has an exponent varying from 1.0 to 1.4 - a range of values that is compatible with existing experimental data. Consequently, existing experiments cannot rule out the possibility of a complete self-preserving solution with a $K \sim t^{-1}$ asymptotic power law decay at high Reynolds numbers. In fact, this asymptotic decay law was found recently in the experiments of Walker (1986).

(4) Since the assumption of complete self-preservation requires that $G$ be constant - and since for high-Reynolds-number isotropic turbulence $\frac{7}{15} G>2$, whereas for lowReynolds-number isotropic turbulence $\frac{7}{15} G<2-$ it is clear that the entire process of isotropic decay from high Reynolds number initial conditions to the final period of decay cannot be described by the theory. The alternative conclusion drawn by George (1987, 1989) arose from the use of a new scaling for the two-point triple velocity correlation that rendered a time dependent skewness where $S_{K} \sim R_{t}^{-1 / 2}$. However, this type of asymptotic behavior for the skewness wherein $S_{K} \rightarrow 0$ as $R_{t} \rightarrow \infty$ is physically inconsistent since it yields zero energy transfer in the infinite Reynolds number limit.

Within the framework of self-preservation, the physical origin of a $K \sim t^{-1}$ power law decay becomes clear: it is the asymptotic state toward which a high-Reynolds number isotropic turbulence is driven in order to resolve an $O\left(R_{t}^{1 / 2}\right)$ imbalance between vortex stretching and viscous diffusion. The resolution of this imbalance also yields compatibility with Kolmogorov scaling. Results were presented which indicate that the complete selfpreserving solution yields a better description of non-equilibrium isotropic turbulence than the commonly used turbulence models. It is also interesting to note that when the self-preserving assumption is extended to homogeneous shear flow, a productionequals-dissipation equilibrium can occur - preceded by a transient where $K$ and $\epsilon$ grow exponentially - as recently shown by Bernard and Speziale (1990). It thus appears that the theory of self-preservation in homogeneous turbulence has many interesting features that have not yet been fully understood and are worthy of further study. 


\section{Acknowledgements}

The authors are indebted to Dr. P. A. Durbin (CTR, Stanford University) and Dr. N. Fitzmaurice (Case Western Reserve University) for some helpful discussions. 


\section{References}

Batchelor, G. K. 1948 Energy Decay and Self-preserving Correlation Functions in Isotropic Turbulence, Quart. Appl. Math, 6, 97.

Batchelor, G. K. 1953 The Theory of Homogeneous Turbulence, Cambridge University Press.

Batchelor, G. K. and Proudman, I. 1956 The Large Scale Structure of Homogeneous Turbulence, Phil. Trans. Roy. Soc. London A, 248, 369.

Batchelor, G. K. and Townsend, A. A., 1948a Decay of Turbulence in the Final Period, Proc. R. Soc. London, Ser. A, 194, 527.

Batchelor, G. K. and Townsend, A. A., 1948b Decay of Isotropic Turbulence in the Initial Period, Proc. R. Soc. London, Ser. A, 193, 539.

Bernard, P. S., 1985 Energy and Vorticity Dynamics in Decaying Isotropic Turbulence, Int. J. Engrg. Sci. 23, 1037.

Bernard, P. S. and Speziale, C. G. 1990 Bounded Energy States in Homogeneous Turbulent Shear Flow - An Alternative View, ICASE Report No. 90 - 66, NASA Langley Research Center.

Comte-Bellot, G. and Corrsin, S., 1966 The Use of a Contraction to Improve the Isotropy of Grid-Generated Turbulence, J. Fluid Mech., 25, 657.

Comte-Bellot, G. and Corrsin, S. 1971 Simple Eulerian Time Correlation of Fulland Narrow-Band Velocity Signals in Grid-Generated "Isotropic" Turbulence, $J$.

Fluid Mech., 48, 273.

Dryden, J. L. 1943 A Review of the Statistical Theory of Turbulence, Quart. Appl. Math, $1,7$.

George, W. 1987 A Theory of the Decay of Homogeneous Isotropic Turbulence, Technical Report No. 116, Turbulence Research Laboratory, SUNY at Buffalo.

George, W. 1989 The Self Preservation of Turbulent Flows and its Relation to Initial

Conditions and Coherent Structures, in Recent Advances in Turbulence, G. Arndt and W. K. George, eds., Hemisphere.

Guckenheimer, J. and Holmes, P. J. 1986 Nonlinear Oscillations, Dynamical Systems and Bifurcations of Vector Fields, Springer-Verlag.

Hinze, J. O., 1975 Turbulence, McGraw-Hill.

Kistler, A. L. and Vrebalovich, T. 1966 Grid Turbulence at Large Reynolds Numbers, $J$. Fluid Mech., 26, 37. 
Korneyev, A. I. and Sedov, L. I. 1976 Theory of Isotropic Turbulence and Its Comparison with Experimental Data, Fluid Mechanics - Soviet Research, 5, 37.

Launder, B. E. and Spalding, D. B. 1974 The Numerical Computation of Turbulent Flows, Comp. Meth. Appl. Mech. and Engrg., 3, 269.

Lesieur, M. 1990 Turbulence in Fluids, 2nd Edition, Martinus Nijhoff.

Monin, A. S. and Yaglom, A. M. 1975 Statistical Fluid Mechanics: Mechanics of Turbulence, Vol. 2, MIT Press.

Proudman, I. and Reid, W. H. 1954 On the Decay of a Normally Distributed and Homogeneous Velocity Field, Proc. Roy. Soc. Lond., Ser. A, 247, 163.

Pumir, A. and Siggia, E. 1990 Collapsing Solutions to the 3-D Euler Equations, Phys. Fluids A, 2, 220.

Rosen, G. 1981 Incompressible Fluid Turbulence at Large Reynolds Numbers: Theoretical Basis for the $t^{-1}$ Decay Law and the Form of the Longitudinal Correlation Function, J. Math. Phys, 22, 1819.

Saffman, P. G. 1967 The Large Scale Structure of Homogeneous Turbulence, J. Fluid Mech., 27, 581 .

Sedov, L. I. 1944 Decay of Isotropic Turbulent Motions of an Incompressible Fluid, Doklady Akademie Nauk, SSSR, 42, 116.

Speziale, C. G. 1991 Analytical Methods for the Development of Reynolds Stress Closures in Turbulence, Ann. Rev. Fluid Mech., 23, 107.

Uberoi, M. 1963 Energy Transfer in Isotropic Turbulence, Phys. Fluids, 6, 1048.

Van Atta, C. W. and Antonia, R. A. 1980 Reynolds Number Dependence of Skewness and Flatness Factors of Turbulent Velocity Derivatives, Phys. Fluids, 23, 252.

Von Karman, T. and Howarth, L. 1938 On the Statistical Theory of Isotropic Turbulence, Proc. Roy Soc. A, 164, 192.

Walker, M. D. 1986 Laser Doppler Measurements of Grid Turbulence in a Box, Ph. D. Thesis, The Johns Hopkins University.

Walker, M. D. and Corrsin, S. 1985 Laser Doppler Measurements of Grid Turbulence in a Box, Bull. Am. Phys. Soc., 30, 1734.

Warhaft, Z. and Lumley, J. L. 1978 An Experimental Study of the Decay of Temperature Fluctuations in Grid-Generated Turbulence, J. Fluid Mech., 88, 659. 
(a)

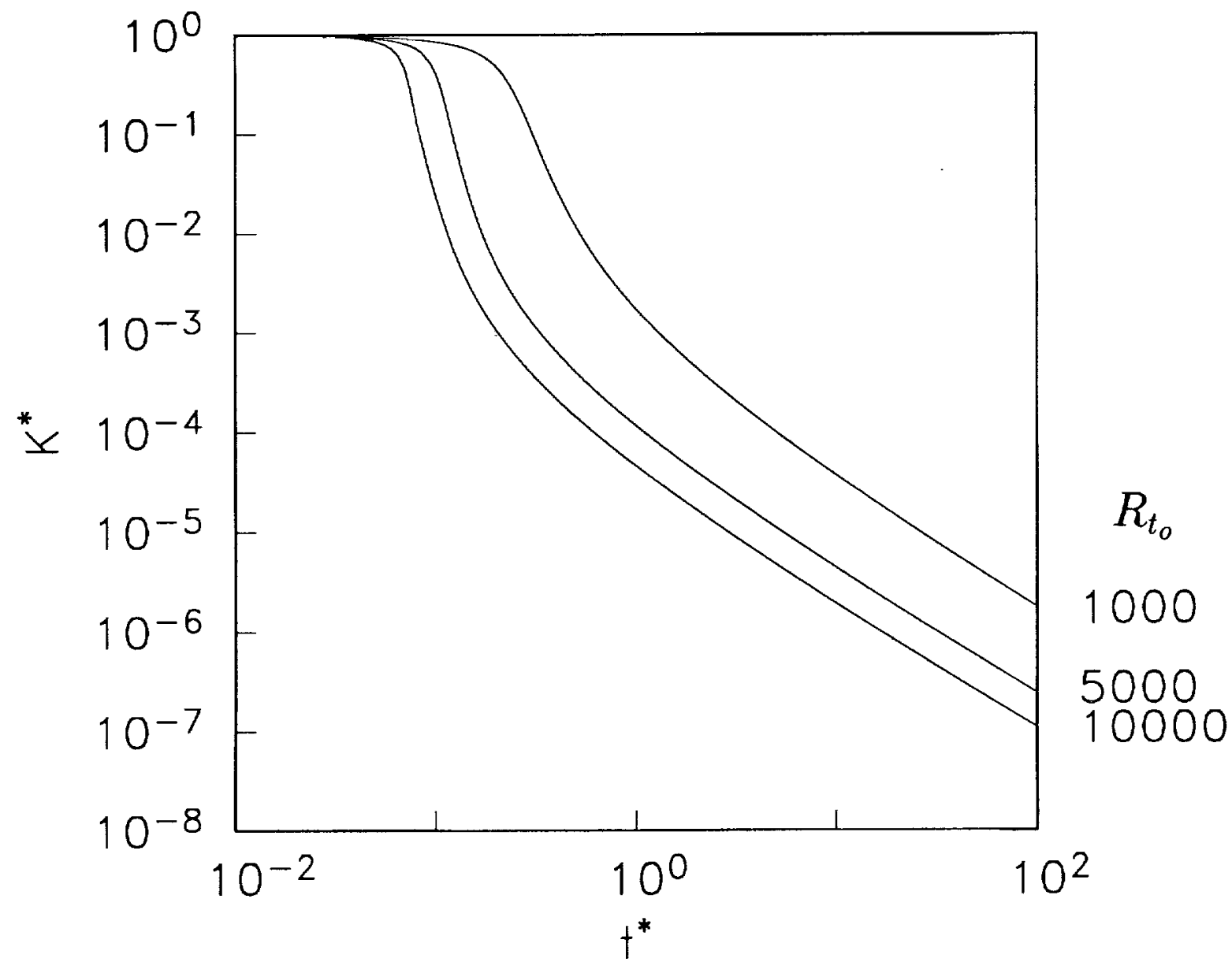

Figure 1. Decay of turbulent kinetic energy in complete self-preserving isotropic turbulence for initial turbulence Reynolds numbers $R_{t_{\circ}}=1000,5000$ and 10,000: (a) $C_{\epsilon_{2}}=$ 1.92 , (b) $C_{\epsilon_{2}}=5$, and (c) $C_{\epsilon_{2}}=8$. 
(b)

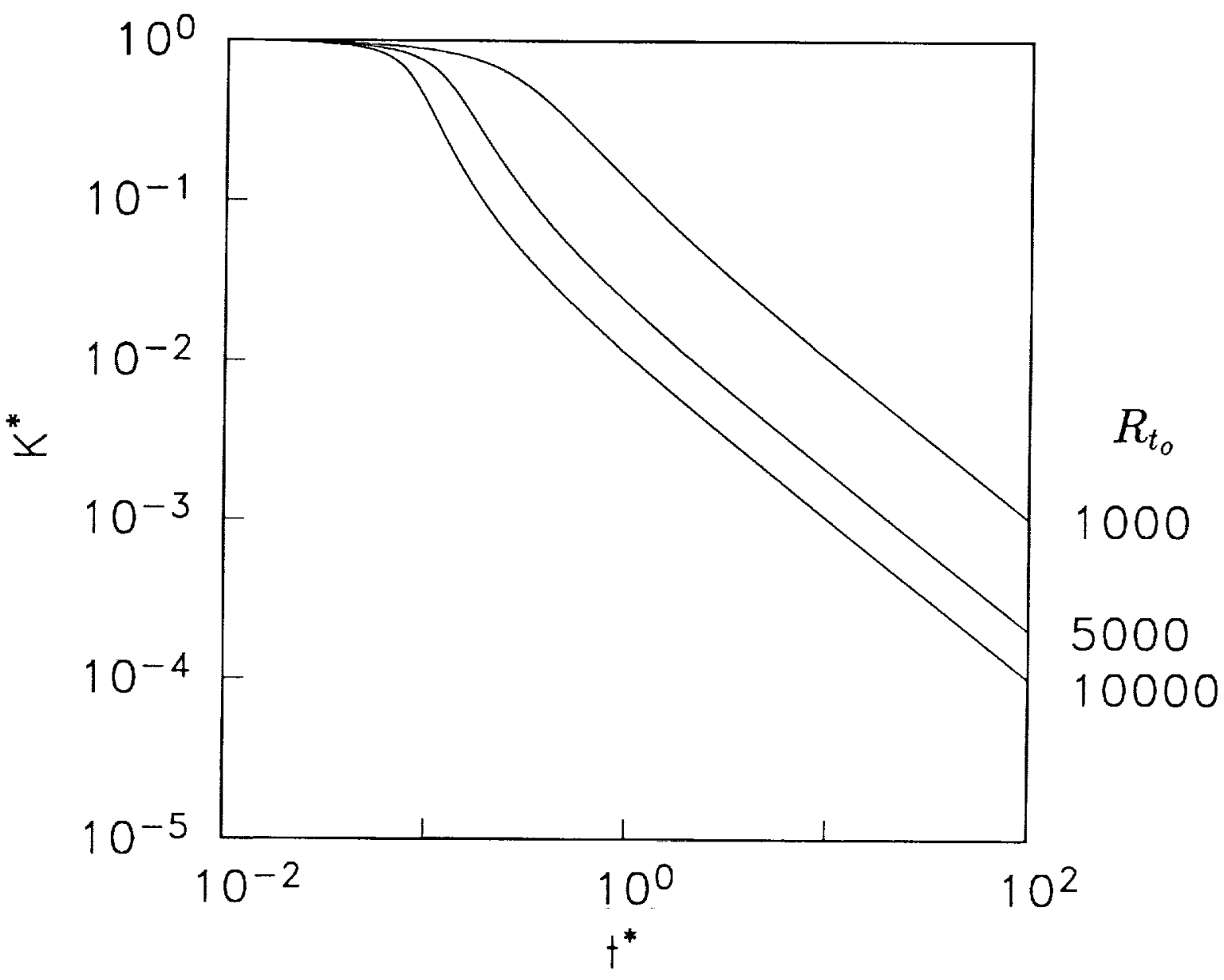

Figure 1. Decay of turbulent kinetic energy in complete self-preserving isotropic turbulence for initial turbulence Reynolds numbers $R_{t_{o}}=1000,5000$ and 10,000: (a) $C_{\epsilon_{2}}=$ 1.92 , (b) $C_{\epsilon_{2}}=5$, and (c) $C_{\epsilon_{2}}=8$. 
(c)

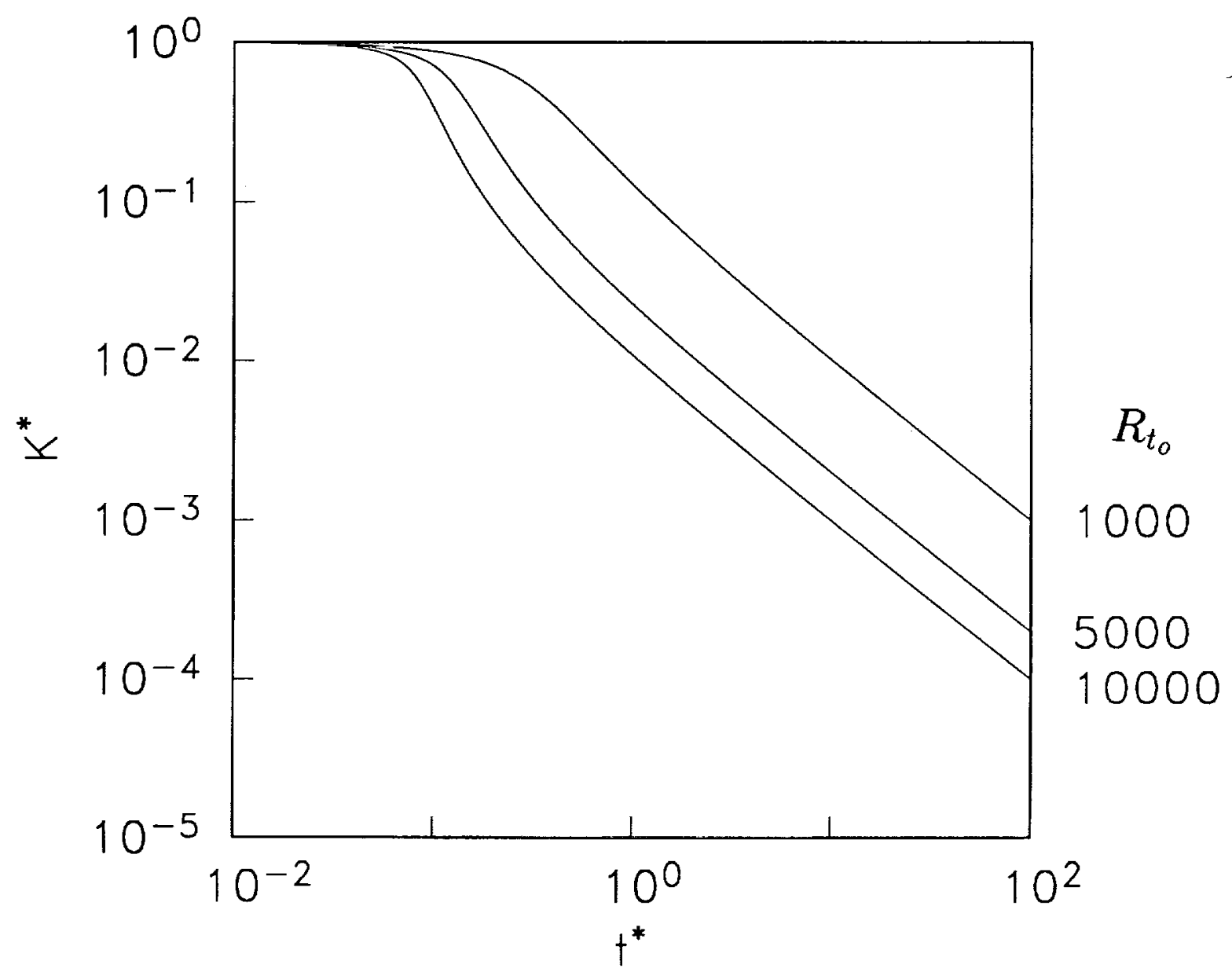

Figure 1. Decay of turbulent kinetic energy in complete self-preserving isotropic turbulence for initial turbulence Reynolds numbers $R_{t_{o}}=1000,5000$ and 10,000: (a) $C_{\epsilon_{2}}=$ $1.92,(\mathrm{~b}) C_{\epsilon_{2}}=5$, and (c) $C_{\epsilon_{2}}=8$. 
(a)

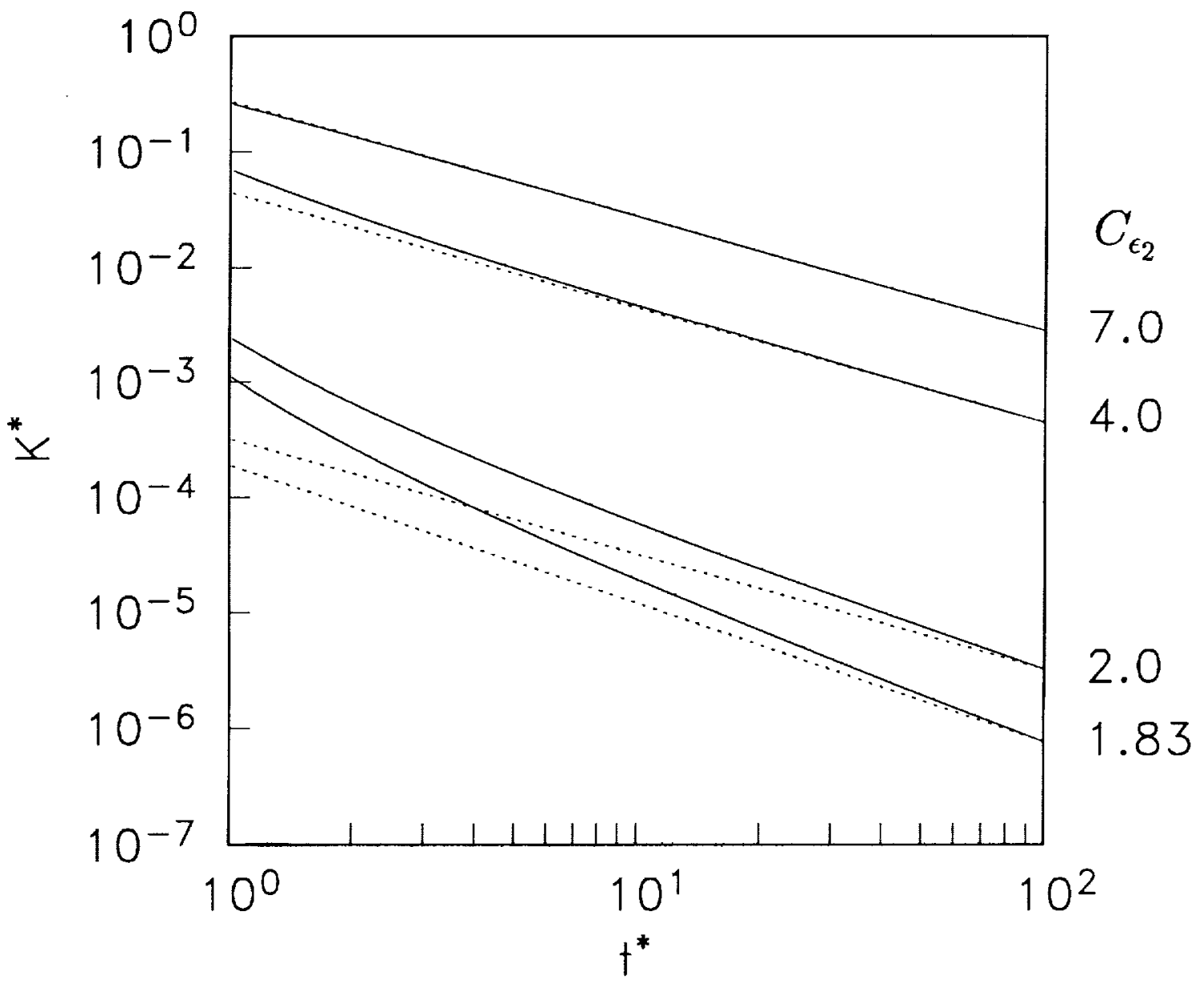

Figure 2. Decay of turbulent kinetic energy for a variety of initial conditions on $G$ $\left(C_{\epsilon_{2}}=\frac{7}{15} G_{0}\right)$ : - - self-preserving solution; - - asymptotic solution $K \sim t^{-\alpha}$ where $\alpha=\left(C_{\epsilon_{2}}-1\right)^{-1}$ for $C_{\epsilon_{2}}<2$ and $\alpha=1$ for $C_{\epsilon_{2}} \geq 2$. (a) $R_{t_{o}}=1000$, (b) $R_{t_{o}}=10,000$ and (c) $R_{t_{o}}=100,000$. 
(b)

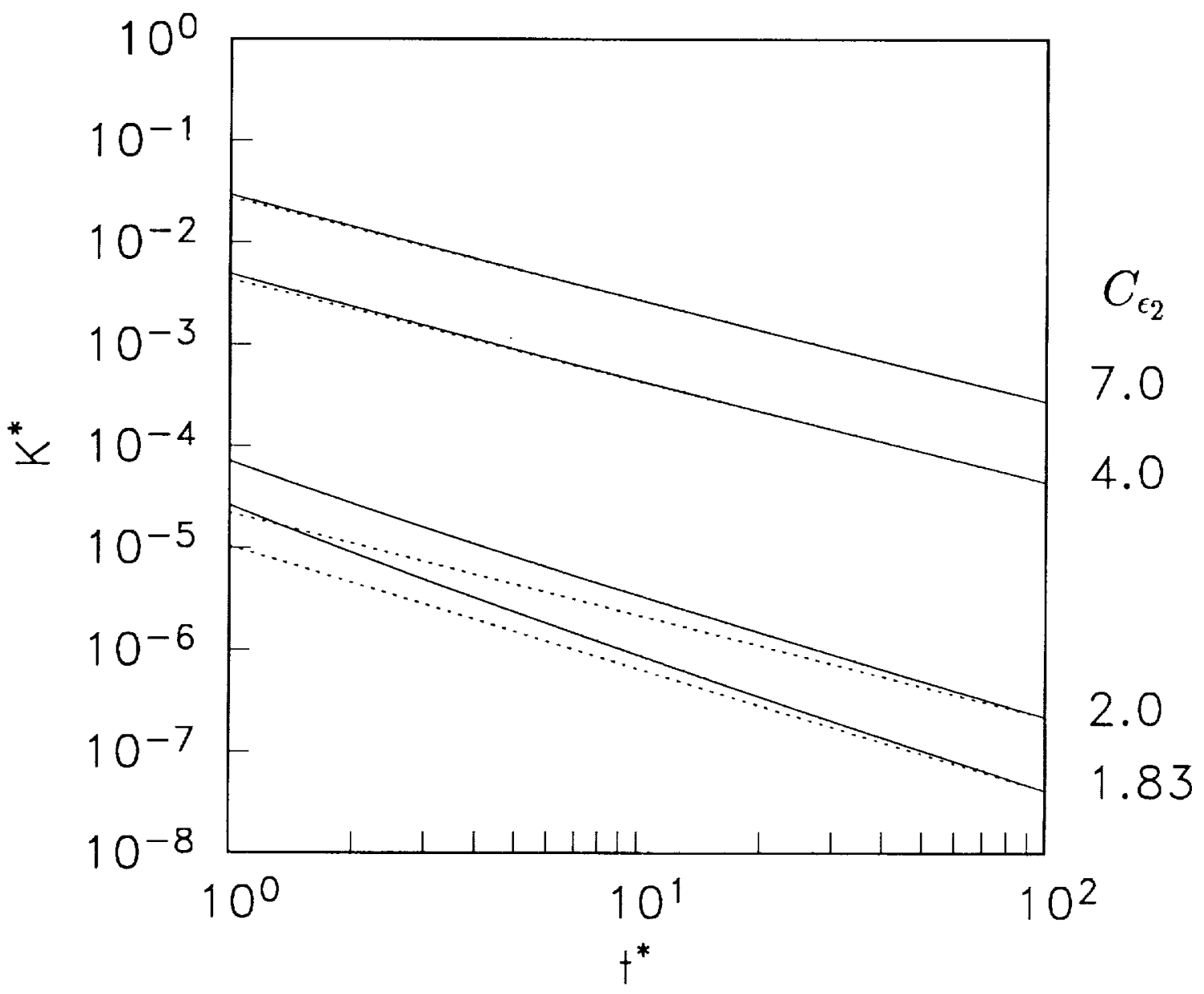

Figure 2. Decay of turbulent kinetic energy for a variety of initial conditions on $G$ $\left(C_{\epsilon_{2}}=\frac{7}{15} G_{0}\right)$ : - self-preserving solution; - - asymptotic solution $K \sim t^{-\alpha}$ where $\alpha=\left(C_{\epsilon_{2}}-1\right)^{-1}$ for $C_{\epsilon_{2}}<2$ and $\alpha=1$ for $C_{\epsilon_{2}} \geq 2$. (a) $R_{t_{o}}=1000$, (b) $R_{t_{o}}=10,000$ and (c) $R_{t_{o}}=100,000$. 
(c)

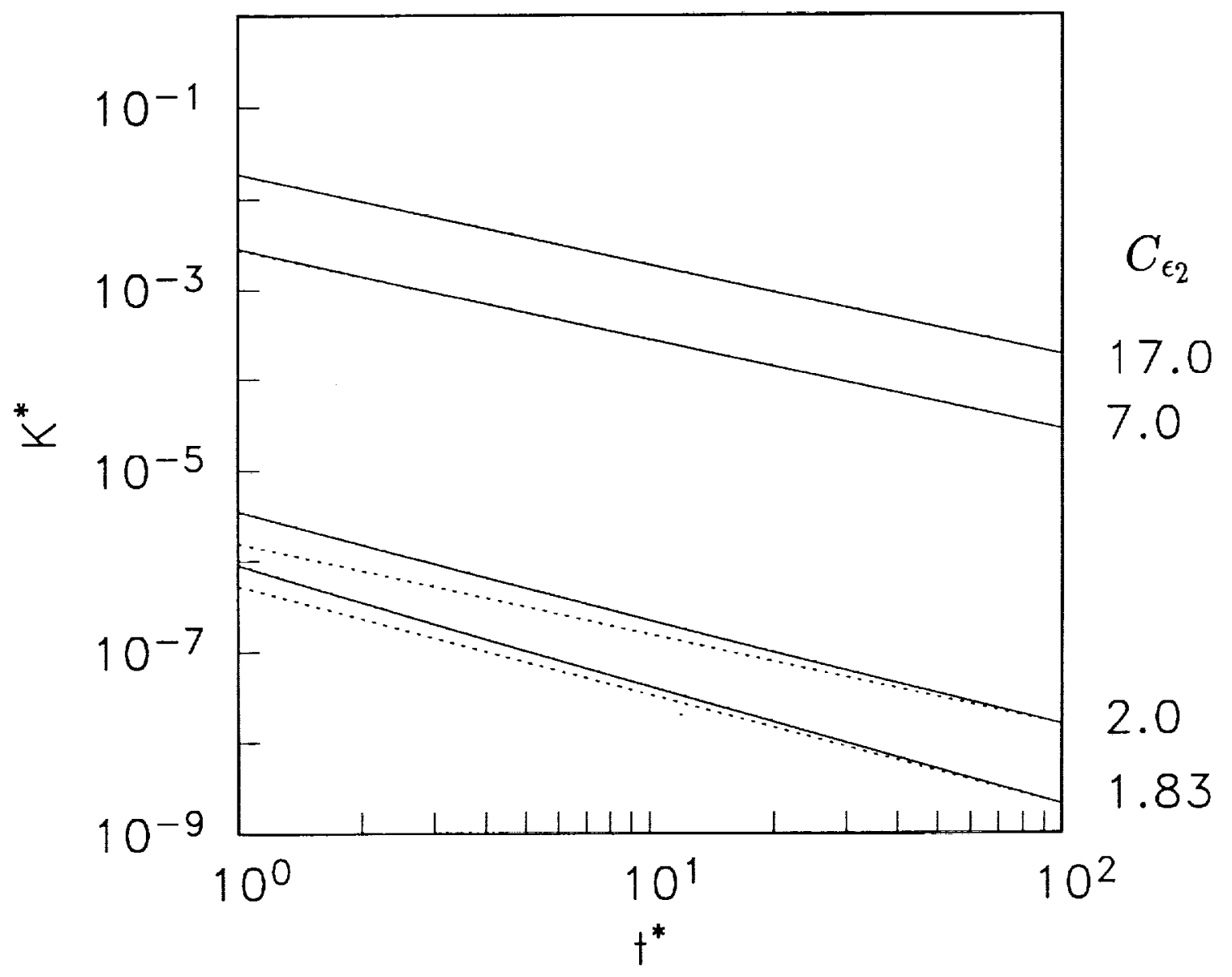

Figure 2. Decay of turbulent kinetic energy for a variety of initial conditions on $G$ $\left(C_{\epsilon_{2}}=\frac{7}{15} G_{0}\right):-$ self-preserving solution; - - asymptotic solution $K \sim t^{-\alpha}$ where $\alpha=\left(C_{\epsilon_{2}}-1\right)^{-1}$ for $C_{\epsilon_{2}}<2$ and $\alpha=1$ for $C_{\epsilon_{2}} \geq 2$. (a) $R_{t_{o}}=1000$, (b) $R_{t_{o}}=10,000$ and (c) $R_{t_{o}}=100,000$. 


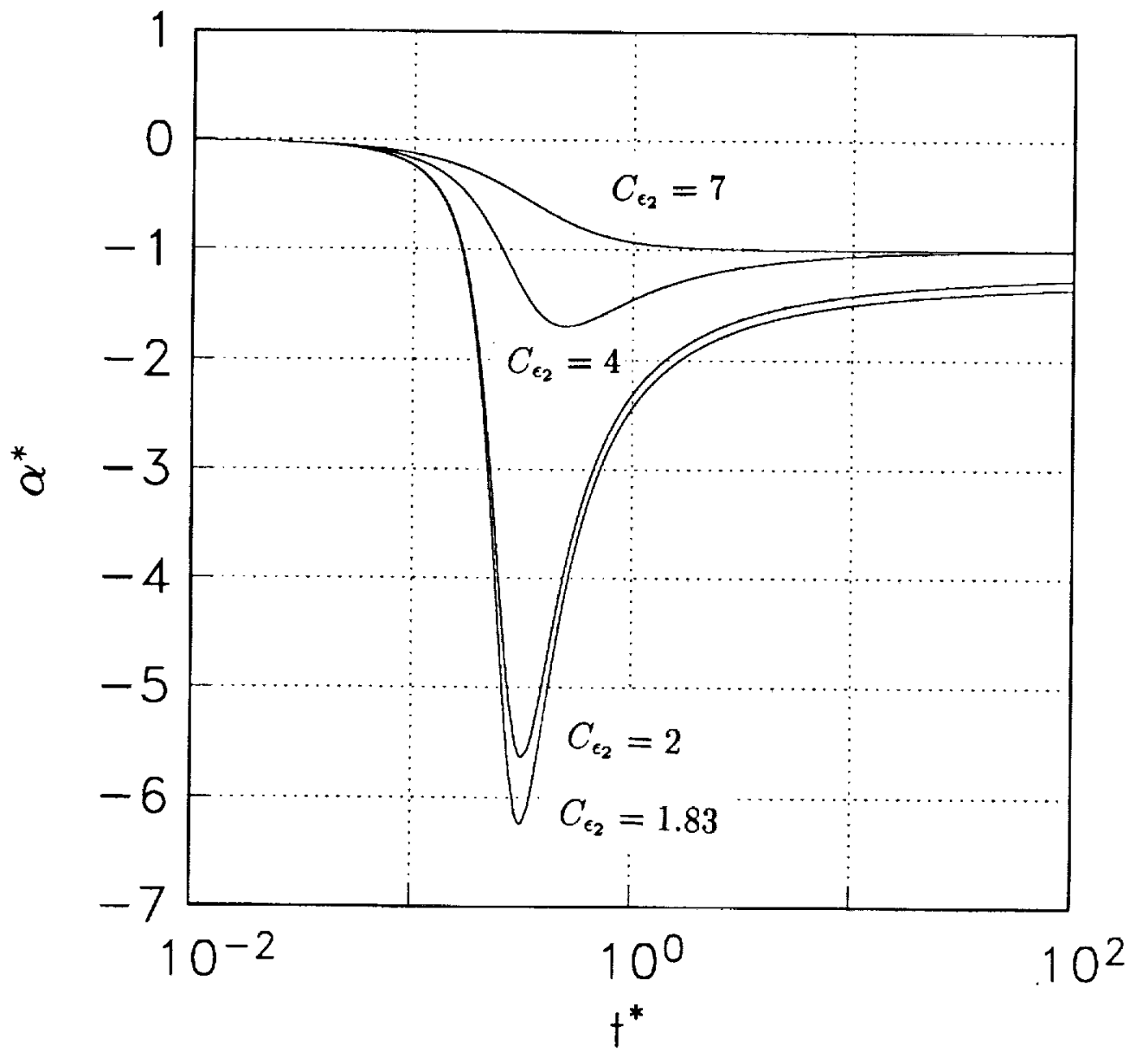

Figure 3. Time evolution of the exponent of the decay law for a variety of initial conditions on $G\left(C_{\epsilon_{2}}=\frac{7}{15} G_{0}\right)$. 


$$
\begin{aligned}
-R_{t_{o}} & =100 \\
--R_{t_{o}} & =200 \\
--R_{t_{o}} & =400
\end{aligned}
$$

(a)

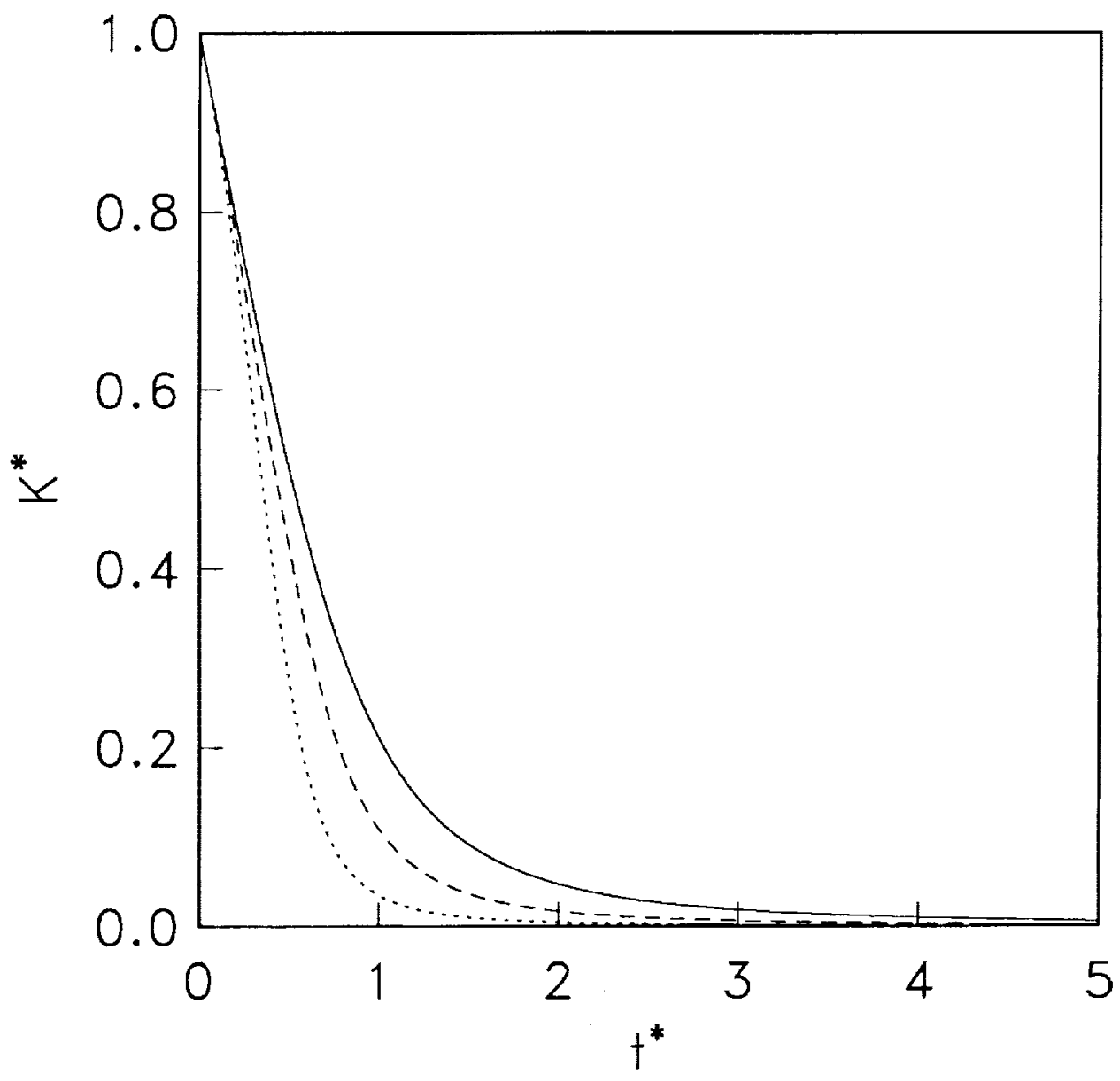

Figure 4. Decay of self-preserving isotropic turbulence for $C_{\epsilon_{2}}=1.83$ and $R_{t_{o}}=100,200$ and 400: (a) turbulent kinetic energy, and (b) turbulent dissipation rate. 


$$
\begin{aligned}
& -R_{t_{o}}=100 \\
& --R_{t_{o}}=200 \\
& -R_{t_{o}}=400
\end{aligned}
$$

(b)

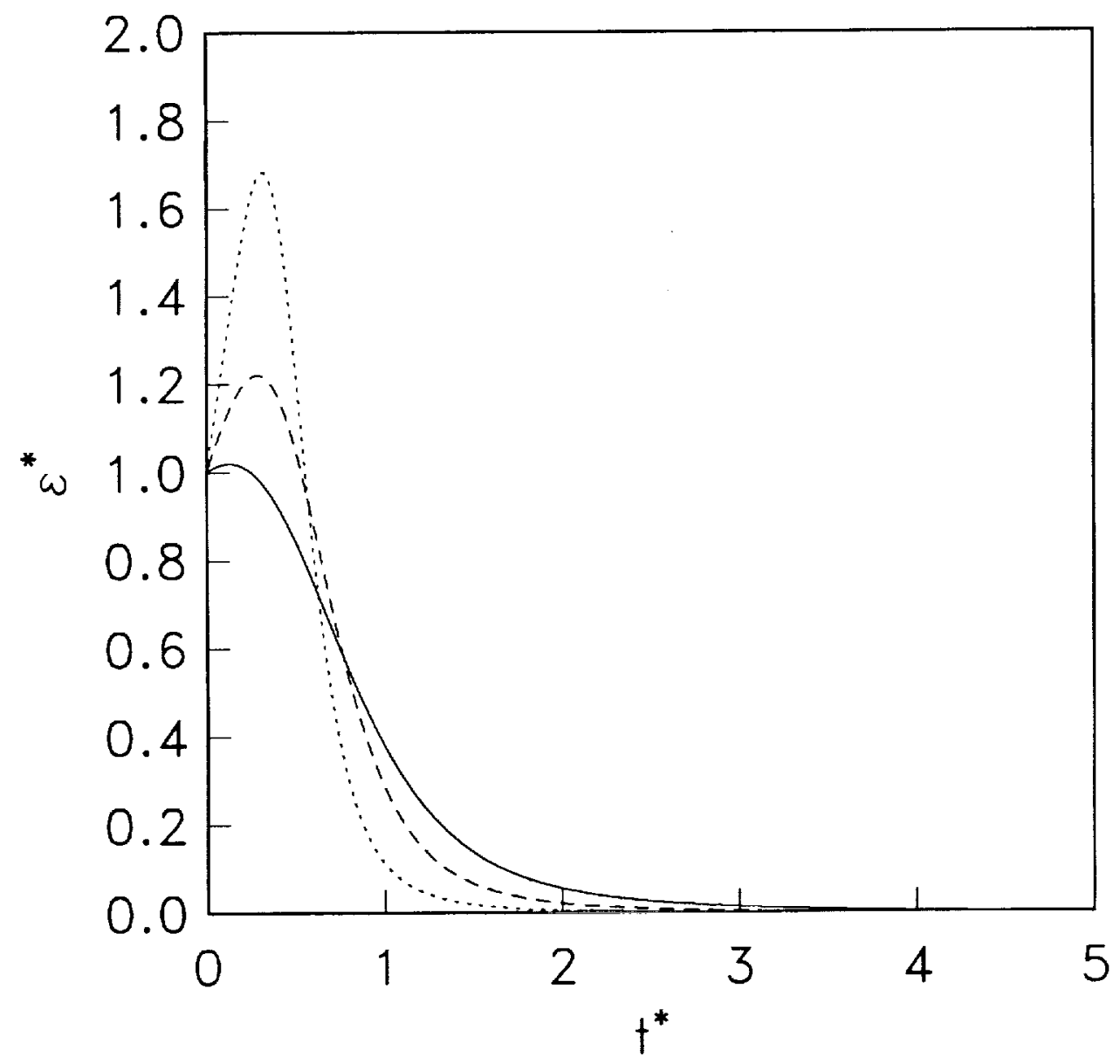

Figure 4. Decay of self-preserving isotropic turbulence for $C_{\epsilon_{2}}=1.83$ and $R_{t_{o}}=100,200$ and 400: (a) turbulent kinetic energy, and (b) turbulent dissipation rate. 


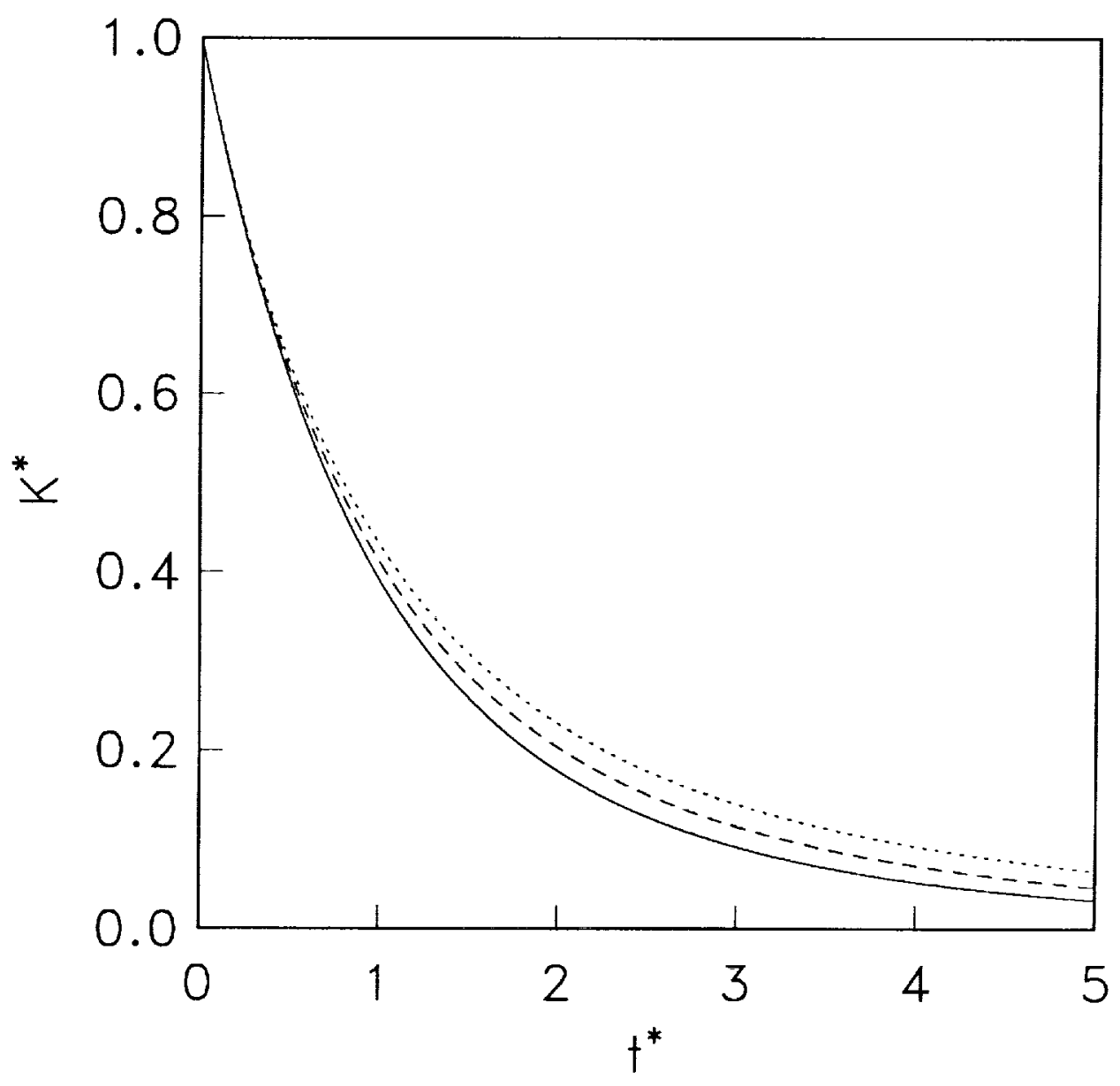

Figure 5. Self-preserving isotropic turbulence at low turbulence Reynolds numbers $\left(C_{\epsilon_{2}}=\right.$ 1.4) :- $R_{t_{o}}=1 ;--R_{t_{o}}=0.25 ;-$ - Eq. (31). 


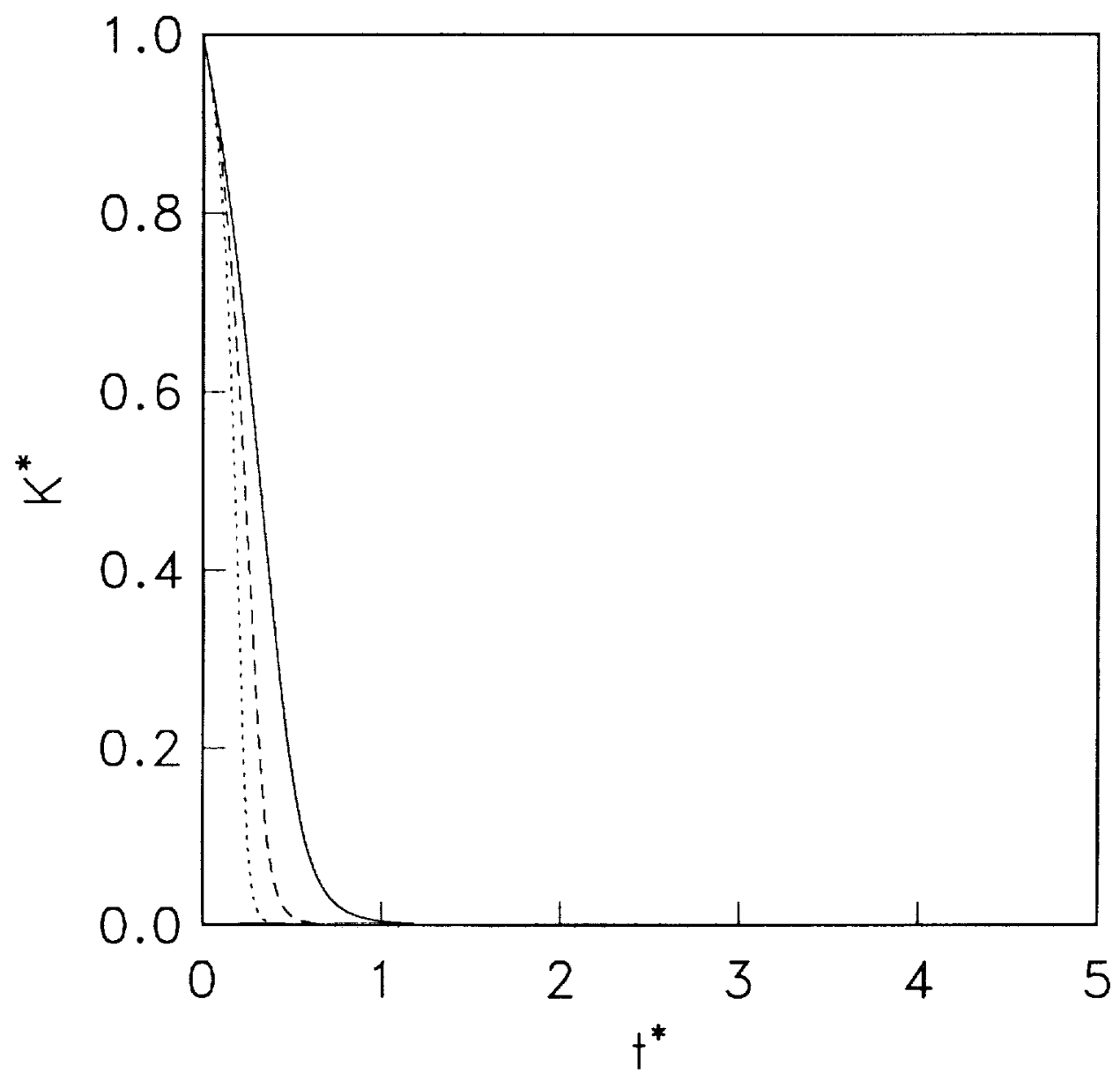

Figure 6. Decay of turbulent kinetic energy in self-preserving isotropic turbulence for $C_{\epsilon_{2}}=1.4:-R_{t_{o}}=200 ;-R_{t_{o}}=500 ;-R_{t_{o}}=1000$. 


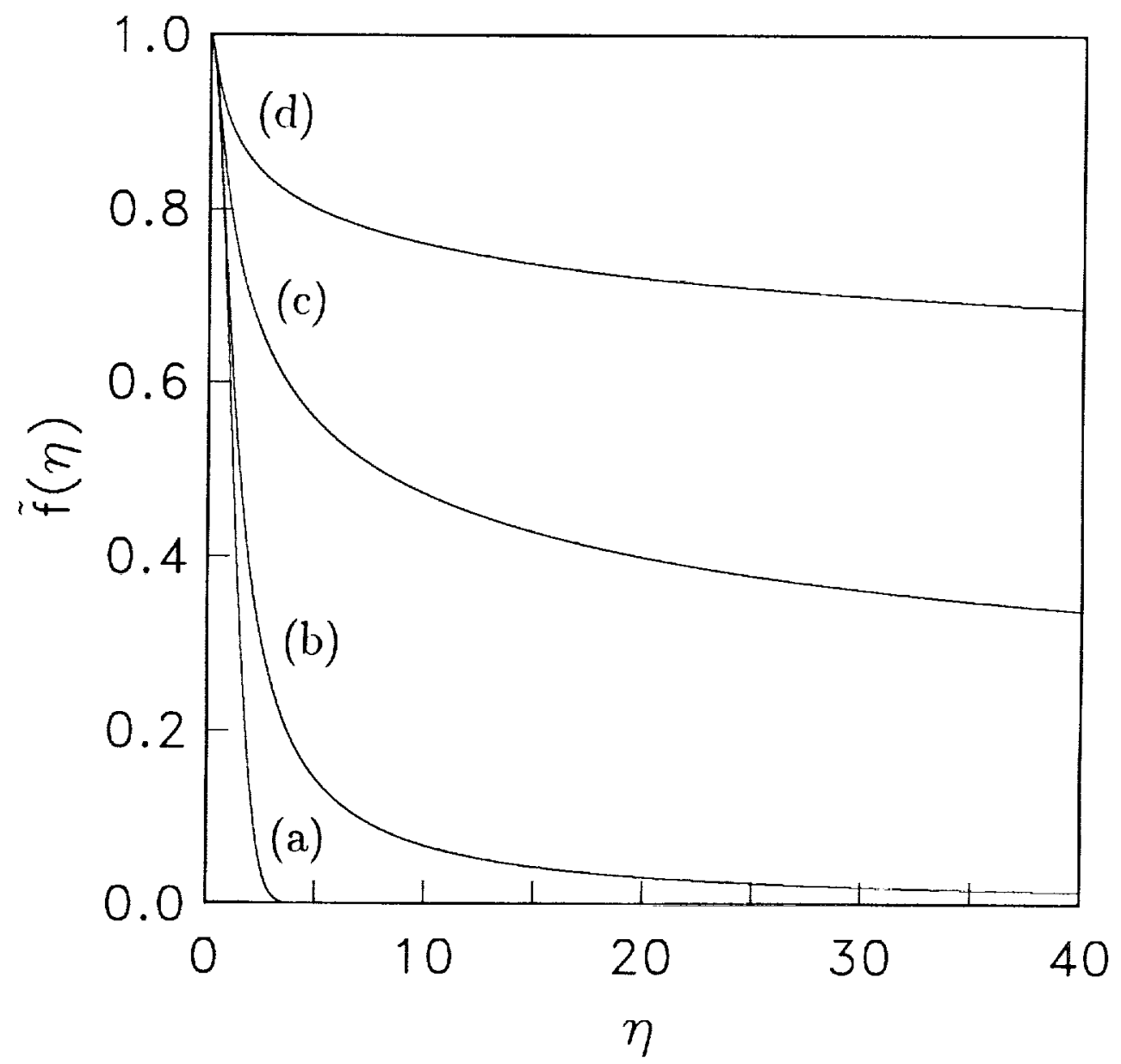

Figure 7. Sedov solution for the two-point double longitudinal velocity correlation: (a) $G_{0}=3$, (b) $G_{0}=6$, (c) $G_{0}=20$, and (d) $G_{0}=60$. 


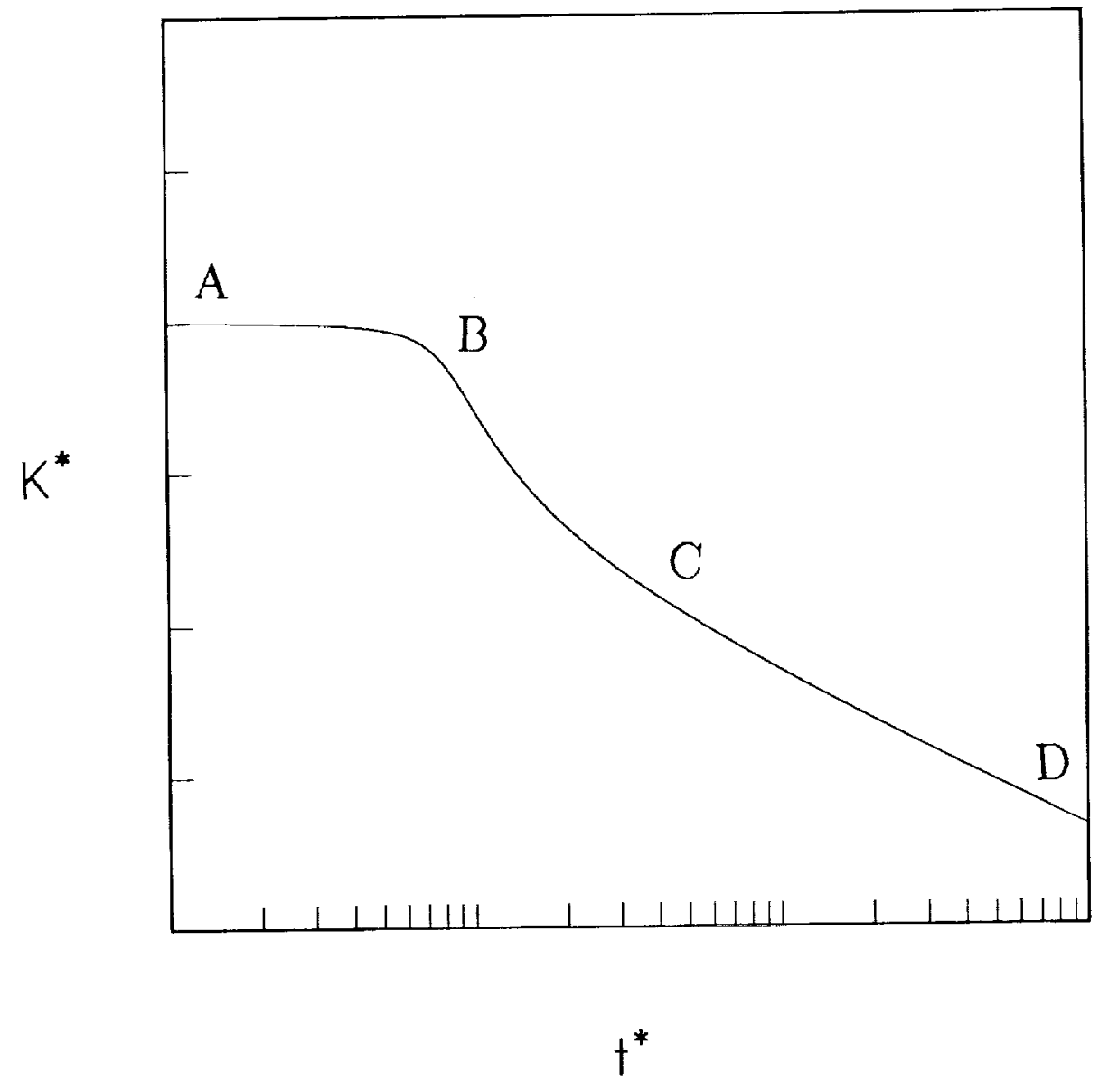

Figure 8. Schematic of the complete self-preserving solution for the decay of turbulent kinetic energy at high Reynolds numbers $\left(R_{t_{o}}>R_{t_{\infty}}\right)$. 


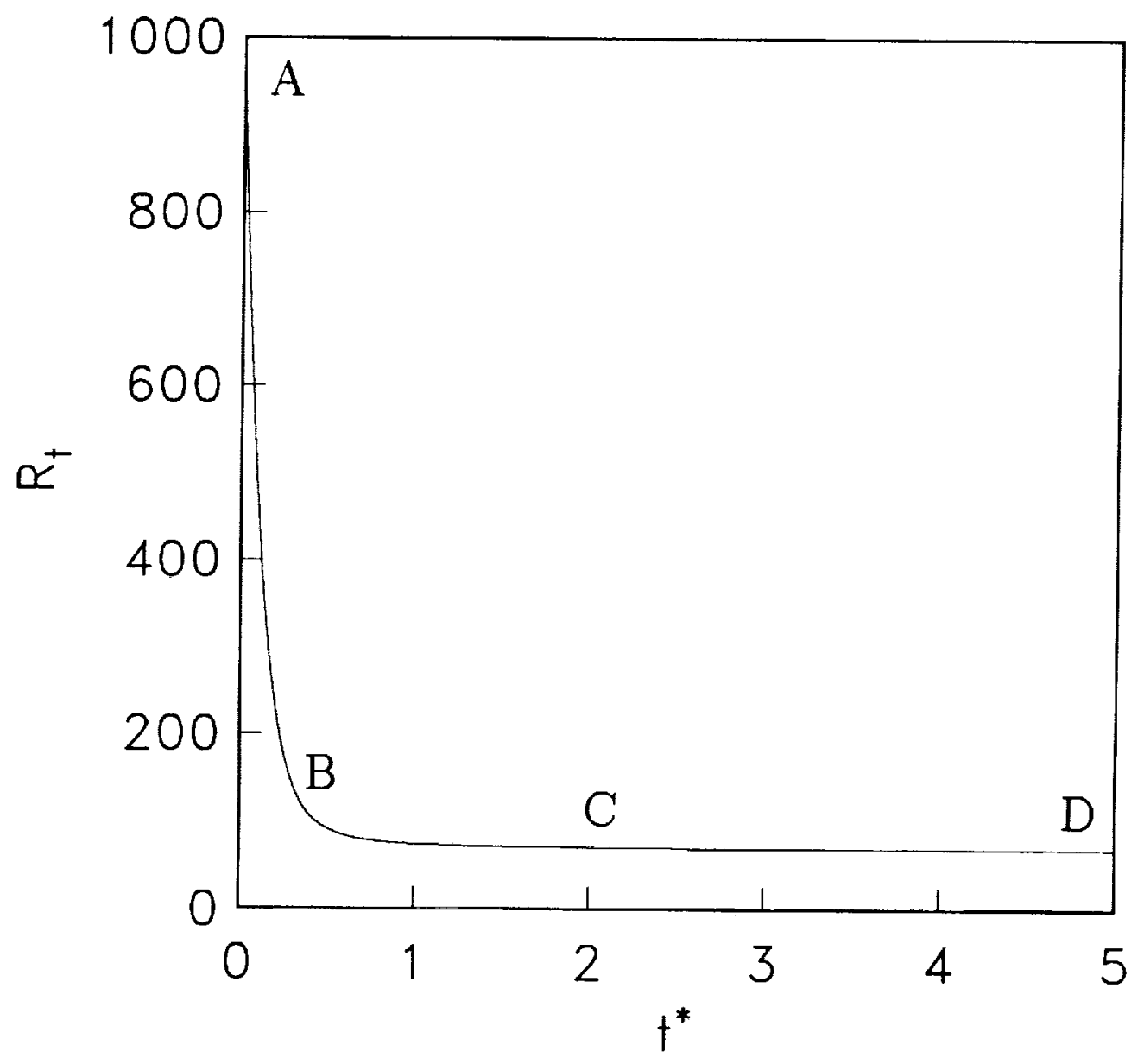

Figure 9. Decay of the turbulence Reynolds number in complete self-preserving isotropic turbulence: $R_{t_{o}}=1000, C_{\epsilon_{2}}=4.5$. 
(a)

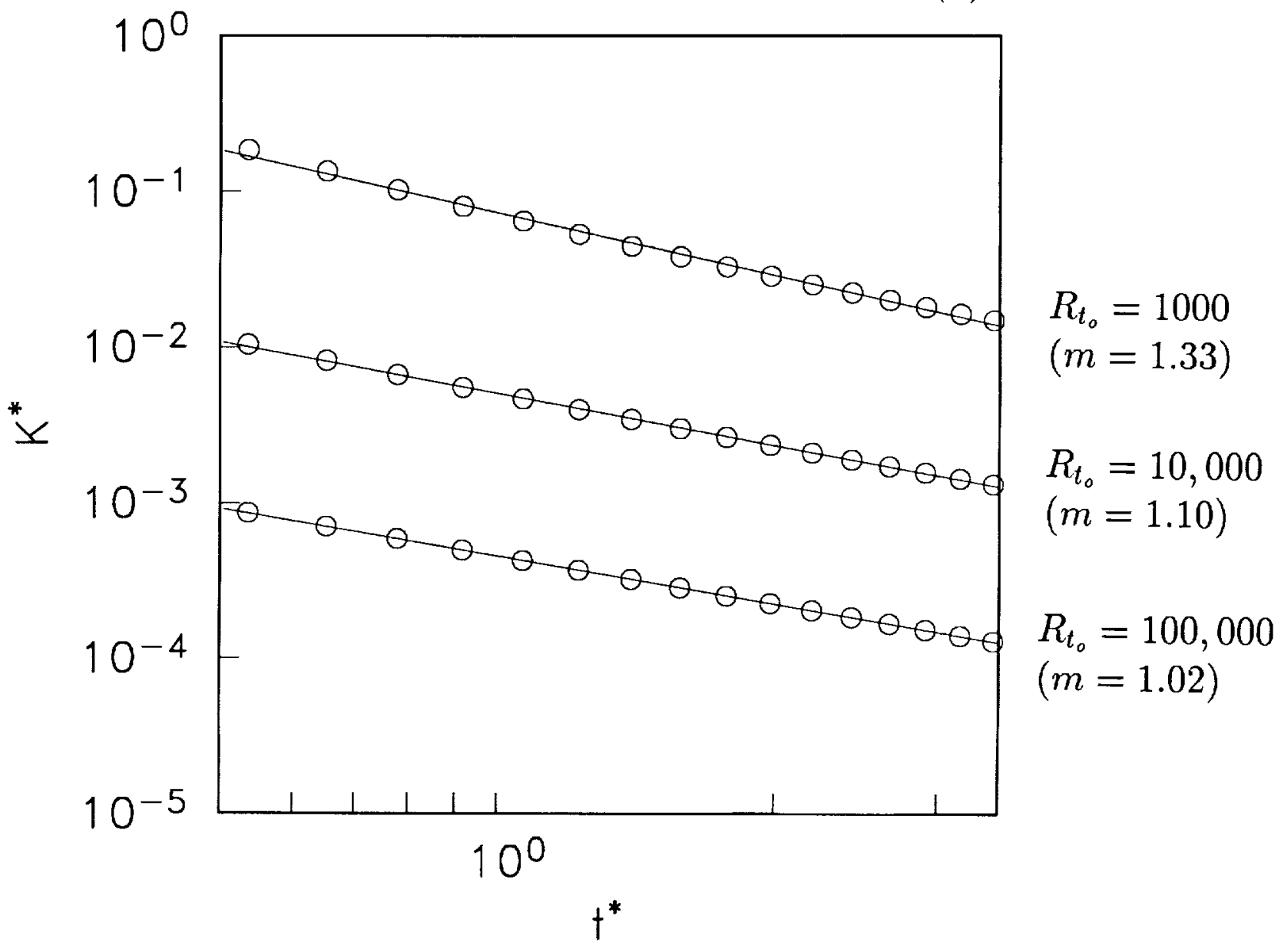

Figure 10. Decay of turbulent kinetic energy: $\circ$ Self-preserving solution; $-K \propto t^{-m}$. (a) $C_{\epsilon_{2}}=4$, (b) $C_{\epsilon_{2}}=4.5$, and (c) $C_{\epsilon_{2}}=5$. 
(b)

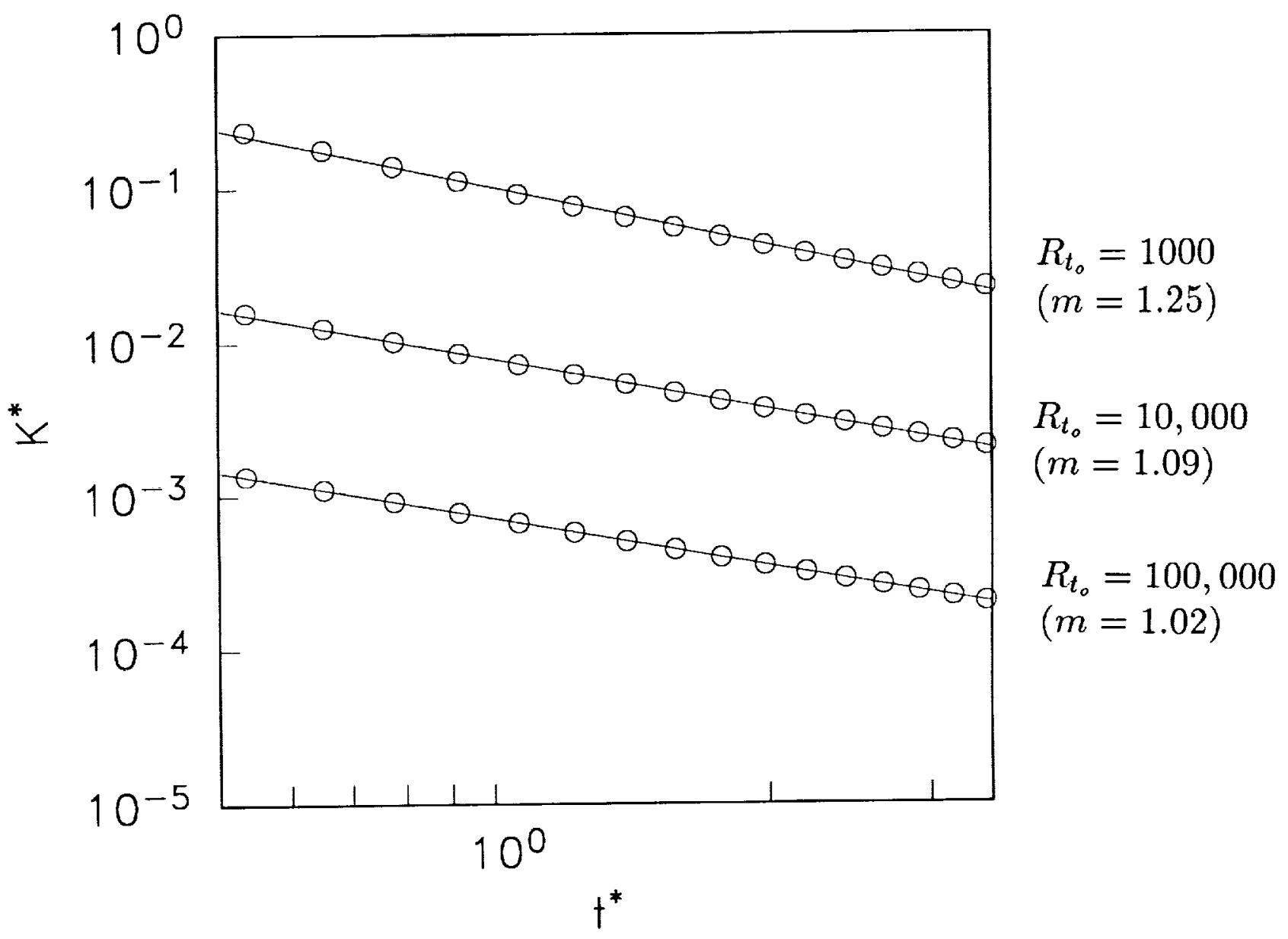

Figure 10. Decay of turbulent kinetic energy: ० Self-preserving solution; $-K \propto t^{-m}$. (a) $C_{\epsilon_{2}}=4$, (b) $C_{\epsilon_{2}}=4.5$, and (c) $C_{\epsilon_{2}}=5$. 
(c)

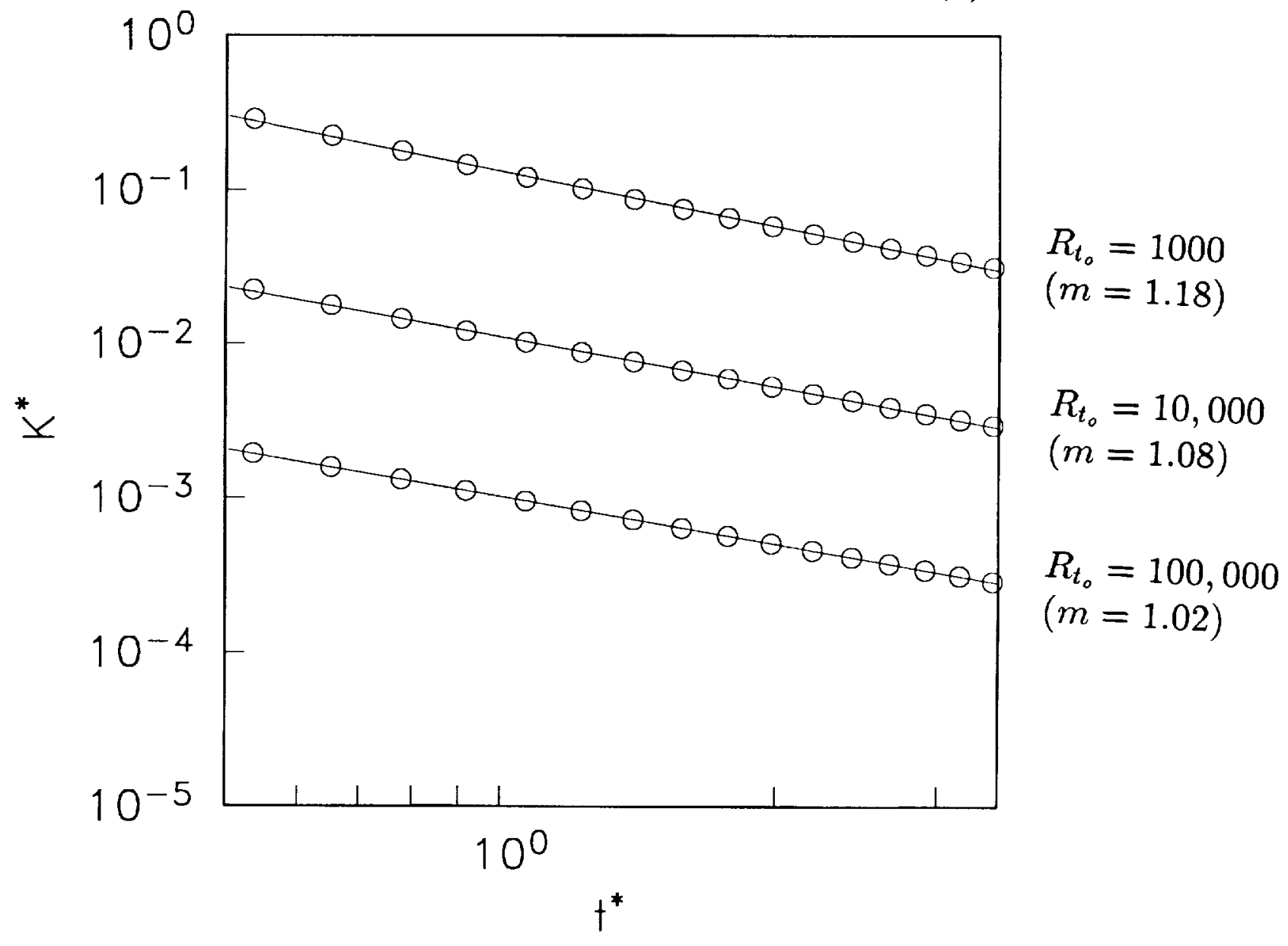

Figure 10. Decay of turbulent kinetic energy: $\circ$ Self-preserving solution; - - $K \propto t^{-m}$. (a) $C_{\epsilon_{2}}=4$, (b) $C_{\epsilon_{2}}=4.5$, and (c) $C_{\epsilon_{2}}=5$. 
(a)

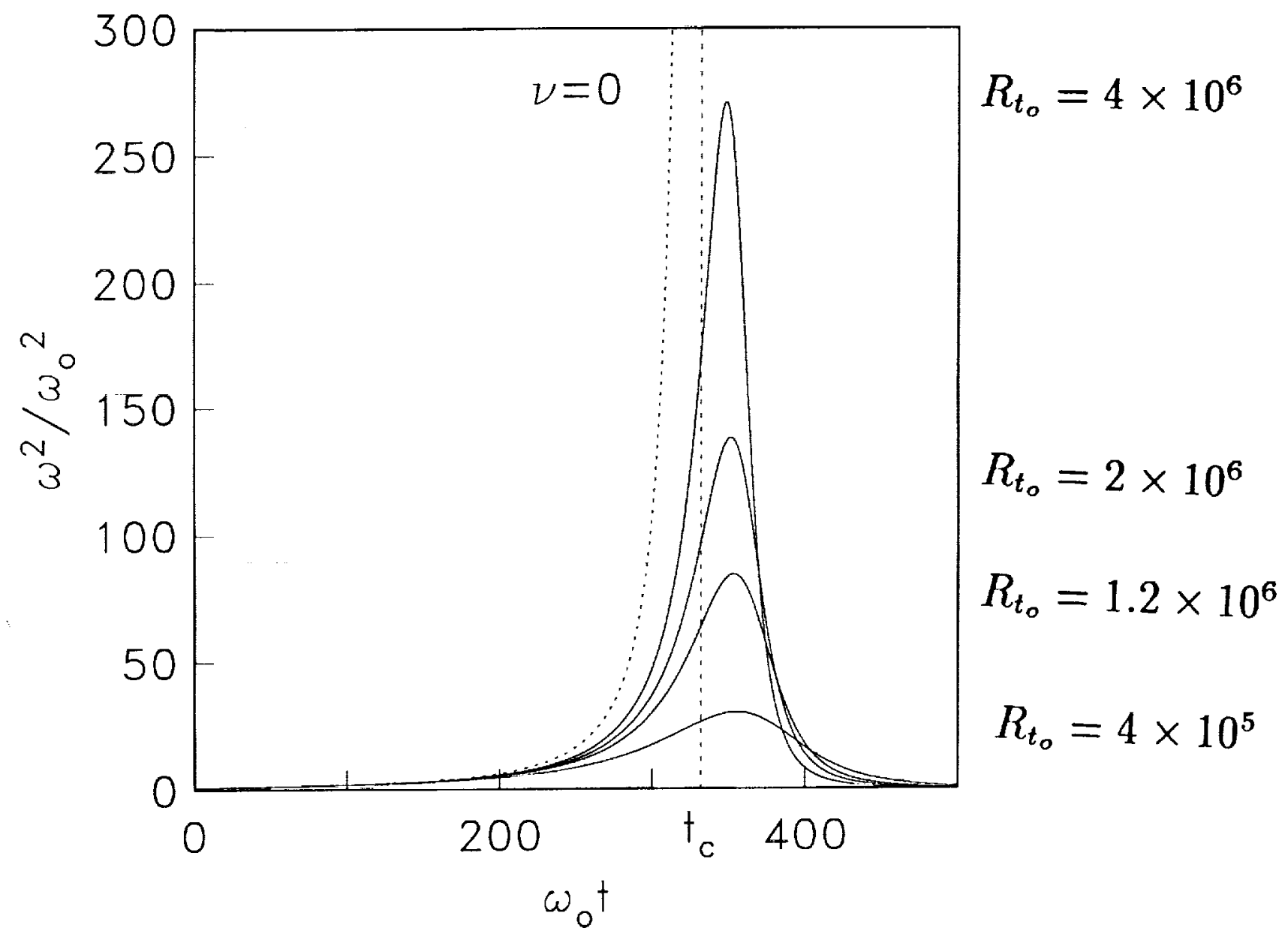

Figure 11. Time evolution of the enstrophy at high turbulence Reynolds numbers: (a) complete self-preserving solution $\left(C_{\epsilon_{2}} \doteq 2\right)$ and (b) schematic from Lesieur (1990) based on EDQNM. 
(b)

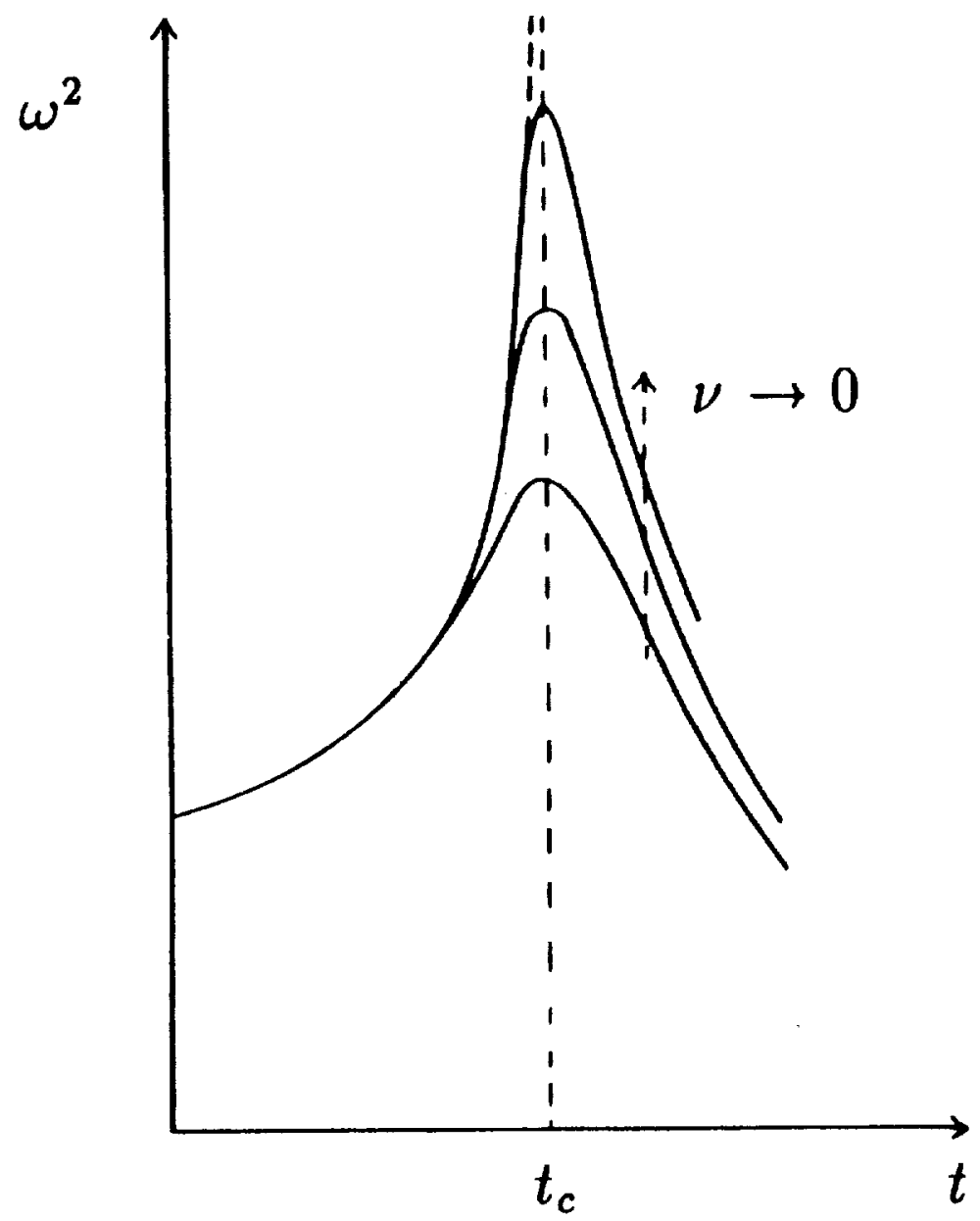

Figure 11. Time evolution of the enstrophy at high turbulence Reynolds numbers: (a) complete self-preserving solution $\left(C_{\epsilon_{2}} \doteq 2\right)$ and (b) schematic from Lesieur (1990) based on EDQNM. 


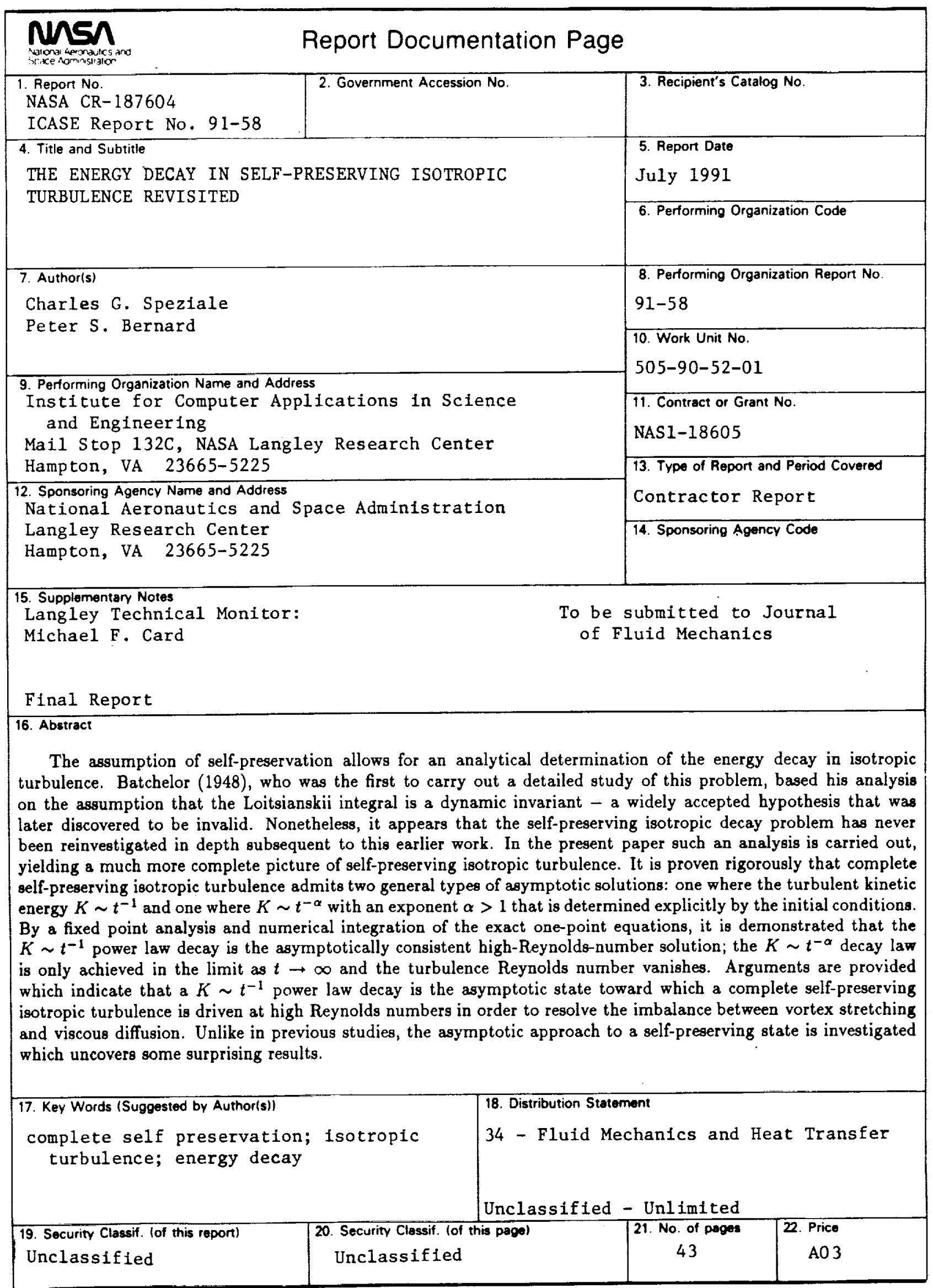

